\title{
Systematic review of the association between exercise tests and patient-reported outcomes in patients with chronic obstructive pulmonary disease
}

\author{
This article was published in the following Dove Press journal: \\ International journal of COPD \\ 22 August 2017 \\ Number of times this article has been viewed
}

\author{
Yogesh Suresh Punekar ${ }^{1}$ \\ John H Riley ${ }^{2}$ \\ Emily Lloyd ${ }^{3}$ \\ Maurice Driessen ${ }^{2}$ \\ Sally J Singh ${ }^{4}$ \\ 'Health Outcomes, GlaxoSmithKline, \\ Uxbridge, ${ }^{2}$ MDC Global Clinical \\ Development UK, Respiratory \\ Research and Development, \\ GlaxoSmithKline, Uxbridge, \\ ${ }^{3}$ Value Evidence and Outcomes, \\ GlaxoSmithKline, Brentford, ${ }^{4}$ Centre \\ for Exercise and Rehabilitation \\ Science, University Hospitals of \\ Leicester NHS Trust, Leicester, UK
}

\begin{abstract}
Introduction: Chronic obstructive pulmonary disease (COPD) is an increasingly common cause of death worldwide. Its cardinal symptoms include breathlessness and severely reduced exercise capacity. Several patient-reported outcome (PRO) measures are used to assess healthrelated quality of life (HRQoL), functional performance, and breathlessness in patients with COPD. Exercise testing is employed to measure functional performance objectively, which is generally believed to impact on overall HRQoL. However, the extent to which commonly used laboratory- and field-based exercise test results correlate with PROs has not been systematically assessed.
\end{abstract}

Materials and methods: A search of Embase, MedLine, and the Cochrane Library identified primary publications in English that reported data on the correlations (Pearson's $r$ or Spearman's $\rho$ ) between the outcomes of exercise tests and HRQoL and breathlessness PROs. Studies reporting on the following tests were included: 6-minute walk test (6MWT), 12MWT, incremental and endurance shuttle walk tests, incremental and endurance cycle ergometer tests, and treadmill tests.

Results: Of 3,205 articles screened, 28 were deemed eligible for inclusion. The most commonly reported HRQoL PRO measure was the St George's Respiratory Questionnaire (13 studies), and the most commonly reported breathlessness PRO measure was the Baseline Dyspnea Index (six studies). The St George's Respiratory Questionnaire appears to correlate very weakly to moderately with the 6MWT, and breathlessness PROs appear to be moderately to strongly associated with 6MWT outcomes. Across all studies, the 6MWT was the most commonly reported exercise test. Very few publications reporting associations between other exercise tests and PRO measures were found.

Conclusion: This review found evidence to support the association of 6MWT outcomes with HRQoL and breathlessness PROs. There were limited data showing correlations with the outcomes of other exercise tests. Further work is required to examine the associations between these PROs and exercise test outcomes.

Keywords: exercise tests, COPD, patient-reported outcomes

\section{Introduction}

Chronic obstructive pulmonary disease (COPD) is a leading cause of death worldwide. The prevalence of the disease is projected to increase as the population ages and as exposure to risk factors, such as smoking, continues. ${ }^{1-3}$ COPD is characterized by breathlessness, episodes of exacerbations, and reduced exercise capacity. ${ }^{4}$ Decreased 
exercise capacity can result in reduced ability to perform the activities of daily living, and the resultant inactivity and sedentary lifestyle can further exacerbate exercise-capacity impairment. ${ }^{5,6}$

In clinical practice, spirometry is recommended by the Global Initiative for Chronic Obstructive Lung Disease (GOLD) for the diagnosis of COPD. ${ }^{4}$ However, spirometry is a poor predictor of disability and health-related quality of life (HRQoL) in patients with $\mathrm{COPD},{ }^{7}$ and correlates weakly with dyspnea, exercise capacity, and health status. ${ }^{8-10}$ Functional evaluations, such as exercise tests, are thus recommended in addition to spirometry. However, there is currently no single standard test for the assessment of exercise capacity. Field-based tests routinely used to measure exercise capacity include the 6-minute walk test (6MWT), ${ }^{11}$ $12 \mathrm{MWT},{ }^{12}$ incremental shuttle walk test (ISWT), ${ }^{13}$ and endurance SWT (ESWT). ${ }^{14}$ Laboratory-based assessments allow the researcher to monitor several concomitant physiological variables, such as heart rate, workload performed, and oxygen consumption. Such tests include the incremental cycle ergometer test (ICET), endurance CET (ECET), and treadmill test (TT). It has been demonstrated that COPD and its consequent limitation of daily activity affect the HRQoL of patients. ${ }^{15}$ Exercise tests are designed to reflect a patient's exercise capacity, but how accurately they reflect patients' HRQoL as assessed by patient-reported outcomes (PROs) of HRQoL and breathlessness in individuals with COPD is unclear. While there have been studies in which exercise tests have been included in the validation of new PRO instruments, ${ }^{16,17}$ it has elsewhere been reported that improvements in exercise capacity elicited by rehabilitation do not necessarily correlate with HRQoL PROs in patients with COPD. ${ }^{18}$

Several questions remain regarding the associations between exercise tests and PROs. Which PROs are independent of exercise and which are not? Which, if any, PROs accurately and consistently reflect the ability of a patient with COPD to perform exercise? The purpose of this systematic review was to assess the strength of the available evidence supporting correlations between the outcomes of different exercise tests and the PROs most commonly used to assess HRQoL and breathlessness.

\section{Materials and methods \\ Search strategy}

Literature searches were conducted using Ovid, incorporating MedLine (1948 to January 22, 2013, then updated to include articles from July 23, 2012 to September 13, 2016), Embase
(1974 to January 22, 2013 and July 23, 2012 to September 13, 2016), and the Cochrane Library (to January 22, 2013 and July 23, 2012 to September 12, 2016). Search strings were constructed to identify study publications (including those specific to emphysema and bronchitis) reporting primary data on the outcomes of the following exercise tests in patients with COPD: 6MWT, 12MWT, ISWT, ESWT, ICET, ECET, and TT. The full search strings used have been published previously. ${ }^{19}$

\section{Study selection}

Study selection followed the Preferred Reporting Items for Systematic Reviews and Meta-Analyses (PRISMA) guidelines for performing a systematic literature review. ${ }^{20}$ Publications were initially screened based on titles and abstracts, and full articles were reviewed when their relevance was unclear from the abstract. Publications were excluded if they were review articles, not in English, studied patients with confounding comorbidities (eg, cancers or diabetes), unclear on the precise variables used for regression analysis, or examined an inappropriate intervention (eg, nonbronchodilatory pharmacotherapy or homeopathy). Articles were subsequently included for assessment only if they reported data on the correlations (Pearson's $r$ and/or Spearman's $\rho$ ) between any of the prespecified exercise tests and PRO measures, such as the St George's Respiratory Questionnaire (SGRQ) ${ }^{17}$ total and individual domain scores (while the former is the usual means of reporting SGRQ data, significant correlations may exist within individual domains alone), the 36-item ShortForm Health Survey (SF-36), ${ }^{21}$ the five-domain European QoL questionnaire (EQ-5D), ${ }^{22,23}$ the Chronic Respiratory Disease Questionnaire (CRQ), ${ }^{24}$ the baseline dyspnea index (BDI), ${ }^{25}$ the oxygen-cost diagram (OCD), ${ }^{26}$ the Medical Research Council dyspnea scale (MRC), ${ }^{27}$ and the modified MRC dyspnea scale (mMRC). ${ }^{28} \mathrm{MRC}$ and mMRC correlations were reported in combination, given that they report the same scale on different intervals.

\section{Exercise tests and PRO measures}

Exercise tests and respiratory-related PRO measures The SGRQ is a disease-specific, self-administered questionnaire assessing symptoms, activity, and impacts on health status in COPD and asthma. Lower scores are associated with improved health status. ${ }^{29}$ The CRQ is a comprehensive HRQoL questionnaire specific for individuals with COPD that assesses dyspnea, fatigue, emotional function, and mastery (the feeling of control over the disease and its effects). Each component is scored using a 7-point Likert 
scale, which can be combined to produce a total score of $20-140$, with higher scores indicating improvement. ${ }^{24}$ The BDI questionnaire assesses the severity of dyspnea based on the three components of functional impairment, magnitude of task, and magnitude of effort, which are rated from 0 (very severe) to 4 (no impairment). These scores can be combined to produce a focal score of $0-12$, with higher scores indicating improved health status. ${ }^{25}$ The OCD assesses dyspnea and measures the oxygen requirement of different activity levels. It is scored from 0 to $100 \mathrm{~mm}$, with higher scores indicating greater improvement. ${ }^{26}$ The MRC is a simple questionnaire that evaluates the effect of breathlessness on daily activities by grading patients' perceptions of breathlessness from 1 to 5 , with lower grades indicating less breathlessness and improved health status. ${ }^{30}$ The mMRC is a 5-point version of this questionnaire..$^{31}$

\section{Exercise tests and non-disease-specific PRO measures}

The SF-36 is a generic questionnaire of 36 items, which assesses physical functioning, social functioning, role limitations (physical), role limitations (emotional), emotional wellbeing, mental health, energy and vitality, pain, general health perceptions, and current general health perceptions compared with the previous year to determine the general health status of a patient. Higher scores indicate better health status. ${ }^{32}$ The EQ-5D is another measure that assesses HRQoL across the five dimensions of mobility, self-care, usual activities, pain/discomfort and anxiety/depression. Each dimension is scored from 1 (no problem) to 3 (severe problem). The test also includes a visual analog scale to measure general HRQoL from 0 to 100 , with 100 representing the best health condition. $^{22}$

\section{Inclusion/exclusion criteria and data abstraction}

Owing to a lack of high-quality evidence for associations between these tests and the prespecified PRO measures, we included observational studies in our final analysis in addition to randomized controlled trials. In our literature search, we reviewed articles to identify those presenting Pearson's and/or Spearman's correlations between our stated PRO measures and the most commonly reported exercise test outcomes. Studies reporting lung-function variables only as a percentage of age-, sex-, and body-mass index-predicted values were excluded. Publications involving studies assessing multivariate regressions were also excluded, owing to the multifactorial nature of the statistical approach and the unsuitability of the output for aggregation.
Data were primarily abstracted by a single reviewer. A randomly generated selection of $30 \%$ of all articles was reviewed by a second reviewer in both phases for qualitycontrol purposes. The following outcomes of exercise tests were recorded: distance or stages achieved for the 6MWT, 12MWT, and ISWT, duration of exercise for the ESWT and ECET, and the highest recorded volume of oxygen consumption (peak $\mathrm{VO}_{2}$ ) and maximum workload $\left(\mathrm{W}_{\max }\right)$ for the TT and ICET.

\section{Statistical analysis}

Pearson's and Spearman's correlations between PRO scores and the most commonly reported exercise test outcomes are presented. Pearson's correlations are often used to describe the linear association between two variables when comparing continuous variable data. Spearman's correlations are commonly used to describe the linear association between two sets of ranked (ordinal) data. Correlations are presented as the range of significant values reported in the study publications reviewed. The strength of correlations has been classified according to British Medical Journal guidelines, which regard significant correlation coefficients of $0-0.19$ as very weak, $0.2-0.39$ as weak, $0.4-0.59$ as moderate, $0.6-0.79$ as strong, and $0.8-1$ as very strong. ${ }^{33}$

\section{Results \\ Overview of identified studies}

The PRISMA-compliant search methodology used to identify relevant articles is summarized in Figure 1. Of 3,205 articles screened, 28 were ultimately deemed eligible for inclusion in this review. ${ }^{34-61}$ Table 1 provides a summary of the studies included.

\section{Correlations between exercise test outcomes and patient-reported quality of life-measure outcomes}

\section{St George's Respiratory Questionnaire total score}

In total, 13 study publications reported correlations between the SGRQ total score $\left(\mathrm{SGRQ}_{\text {total }}\right)$ and one or more exercise test outcomes (Table 2, Figure 2). ${ }^{37,41-43,45,46,49,50,52,53,55,59,60}$ Of these, correlations between outcomes from the $\mathrm{SGRQ}_{\text {total }}$ and distance covered in the 6MWT were most commonly reported. These correlations were typically significant, with six articles reporting weak negative Pearson's correlations of $-0.26,{ }^{37}-0.26(P<0.01),{ }^{59}-0.37(P<0.05),{ }^{41}-0.37$ $(P=0.0228),{ }^{49}-0.39(P<0.01),{ }^{53}$ and $-0.39(P=0.01),{ }^{60}$ demonstrating that as distance covered in the 6MWT increases, $\mathrm{SGRQ}_{\text {total }}$ scores decrease, indicating better 


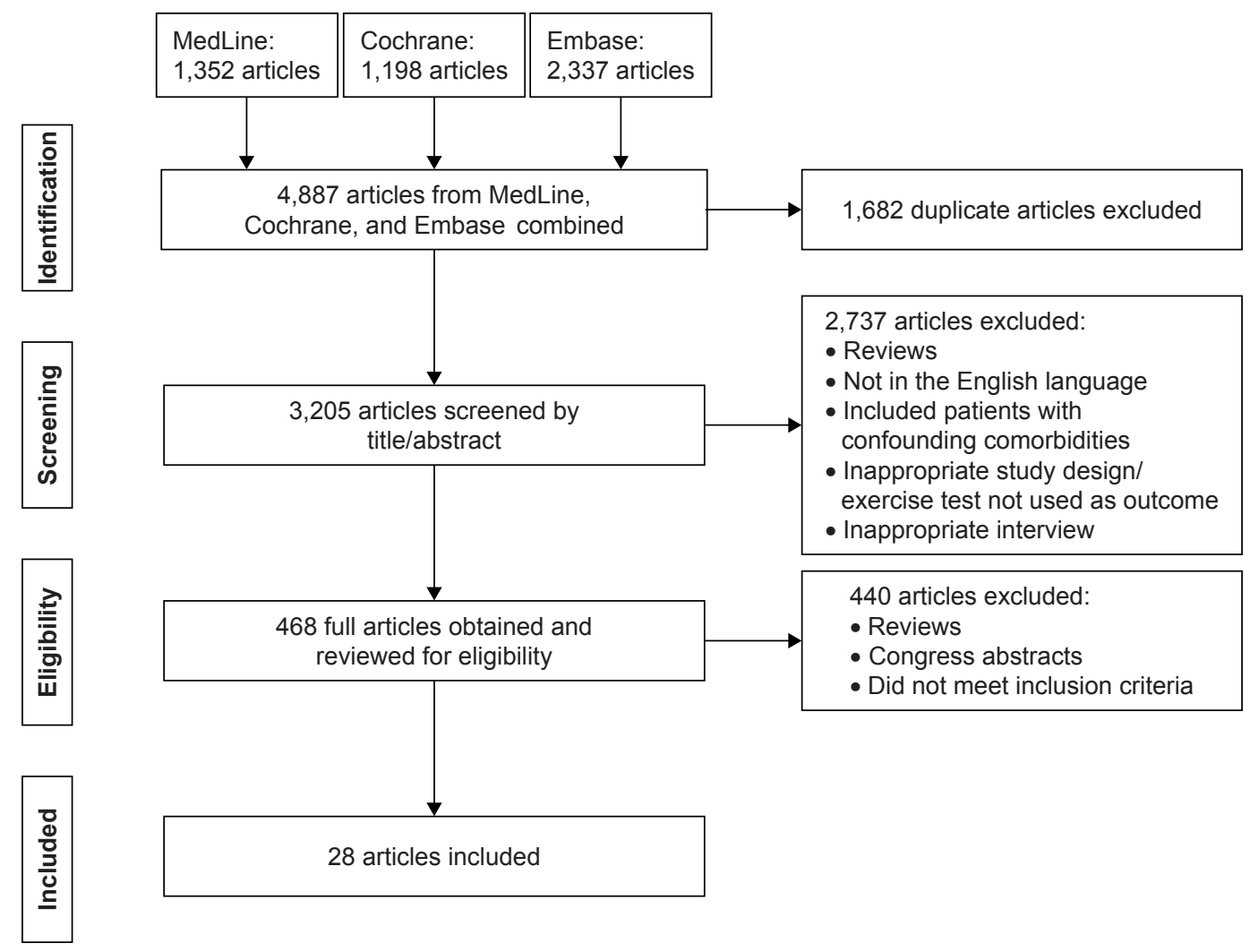

Figure I PRISMA-compliant screening and identification process.

Abbreviation: PRISMA, Preferred Reporting Items for Systematic Reviews and Meta-Analyses.

health status. There were also two studies reporting Spearman's correlations of $-0.27^{42}$ (JP de Torres confirmed this was incorrectly reported as 0.27 in the article) and $-0.56 .^{50}$ Three papers noted nonsignificant associations between the $6 \mathrm{MWT}$ and $\mathrm{SGRQ}_{\text {total }}{ }^{46,52,55}$ Limited data were available for other exercise tests, with moderate correlations given for the ISWT $\left(r=-0.55^{45}, \rho=-0.55^{43}\right)$, weak-moderate correlations for the ICET $(r=-0.29$ to $-0.23,{ }^{37,41} \rho=-0.49$ to $-0.36,{ }^{50}$ depending on the outcome measure obtained $\left[\mathrm{VO}_{2}\right.$ or $\left.\mathrm{W}_{\text {max }}\right]$ ), and moderate Spearman's correlations $\left(\rho=-0.54^{50}\right.$ and $\left.-0.58^{43}\right)$ for the ECET and TT, respectively.

\section{St George's Respiratory Questionnaire activity domain}

Correlations between exercise test outcomes and the SGRQ activity domain score $\left(\mathrm{SGRQ}_{\text {activity }}\right)$ were reported in nine study publications (Table 2). ${ }^{37,43,45,46,50,53,55,59,60}$ Of these, four articles noted significant weak-strong negative correlations between 6MWT distance and $\mathrm{SGRQ}_{\text {activity }}\left(r=-0.35,{ }^{37}-0.36^{53}\right.$ [weak], and $-0.44^{60}$ [moderate]; $\rho=-0.68^{50}$ and an unspecified correlation of $-0.37^{46}$ ). Two further studies found strong correlations between $\mathrm{SGRQ}_{\text {activity }}$ and ISWT outcome
( $r=-0.67^{45}$ and $\rho=-0.62^{43}$ ), with two more finding no significant association for this relationship..$^{55,59}$ One study ${ }^{50}$ reported moderate correlations between $\mathrm{SGRQ}_{\text {activity }}$ and ICET peak $\mathrm{VO}_{2}(\rho=-0.51)$ and strong correlations between $\mathrm{SGRQ}_{\text {activity }}$ and ICET $\mathrm{W}_{\max }(\rho=-0.62)$ and ECET $(\rho=-0.62)$, with a weak correlation for the ICET $\mathrm{W}_{\text {max }}$ found in one other study $(r=-0.31) .{ }^{37}$ Finally, one publication reported moderate correlations between TT peak $\mathrm{VO}_{2}$ and $\mathrm{SGRQ}_{\text {activity }}(\rho=-0.58){ }^{43}$ Again, these negative correlations suggest that as exercise performance increases, $\mathrm{SGRQ}_{\text {activity }}$ scores decrease, indicating improved health status.

\section{St George's Respiratory Questionnaire impact domain}

Correlations between exercise test outcomes and the SGRQ impact domain score $\left(\mathrm{SGRQ}_{\text {impact }}\right)$ were reported in nine articles (Table 2). ${ }^{37,43,45,46,50,53,55,59,60}$ Five studies found weakmoderate correlations between the $\mathrm{SGRQ}_{\text {impact }}$ and the 6MWT $\left(r=-0.22,-0.28,-0.37,,^{37,53,59}\right.$ and $\left.0.4,{ }^{60} \rho=-0.5^{55}\right)$, two found moderate evidence for an association between the $\mathrm{SGRQ}_{\text {impact }}$ and the ISWT $\left(r=-0.53^{45}\right.$ and $\left.\rho=-0.48^{43}\right)$, and there was moderate evidence for an association between the $\mathrm{SGRQ}_{\text {impact }}$ and the ECET $(\rho=-0.50)^{50}$ or TT $(\rho=-0.54) .{ }^{43}$ A further study 


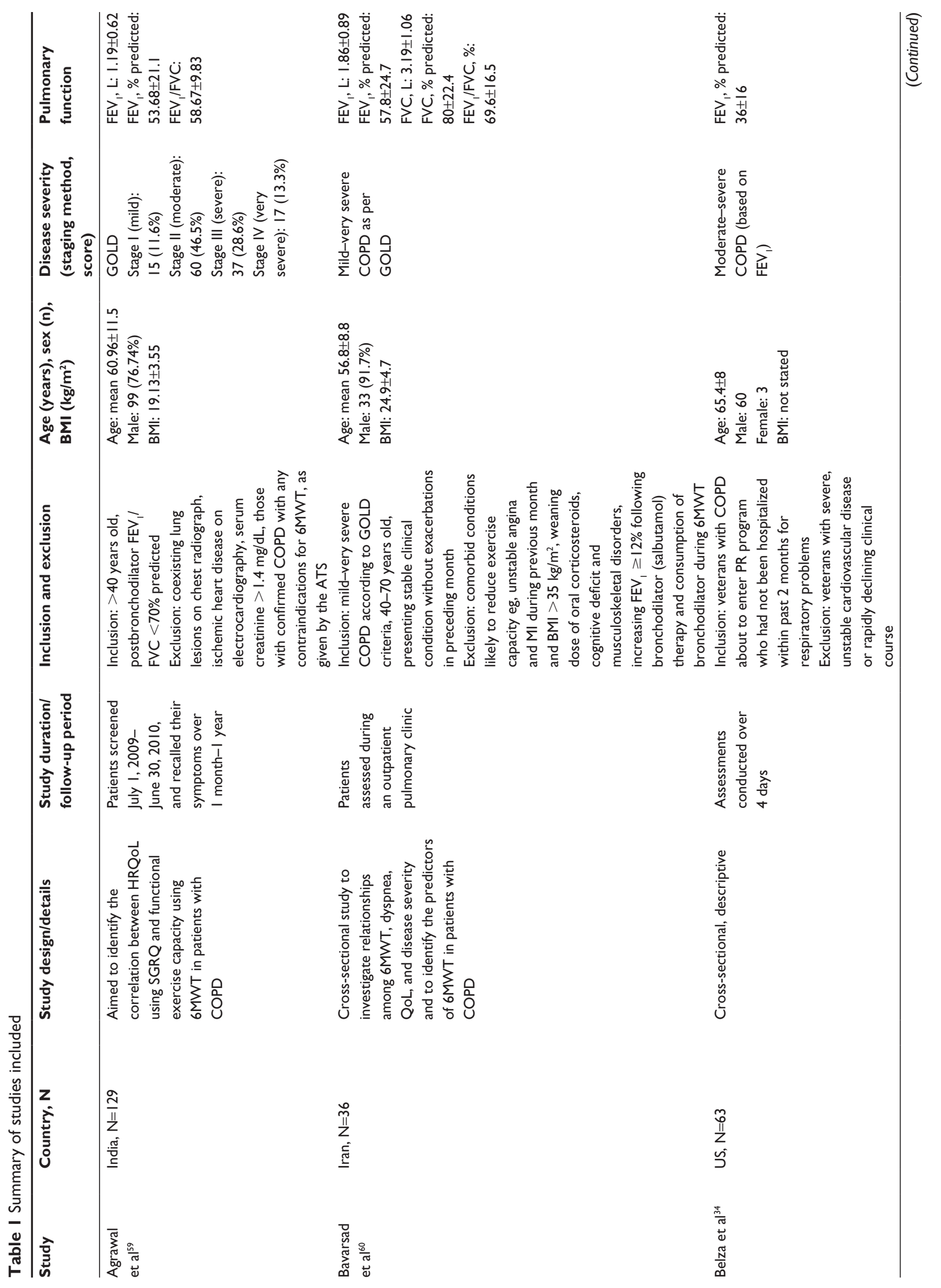




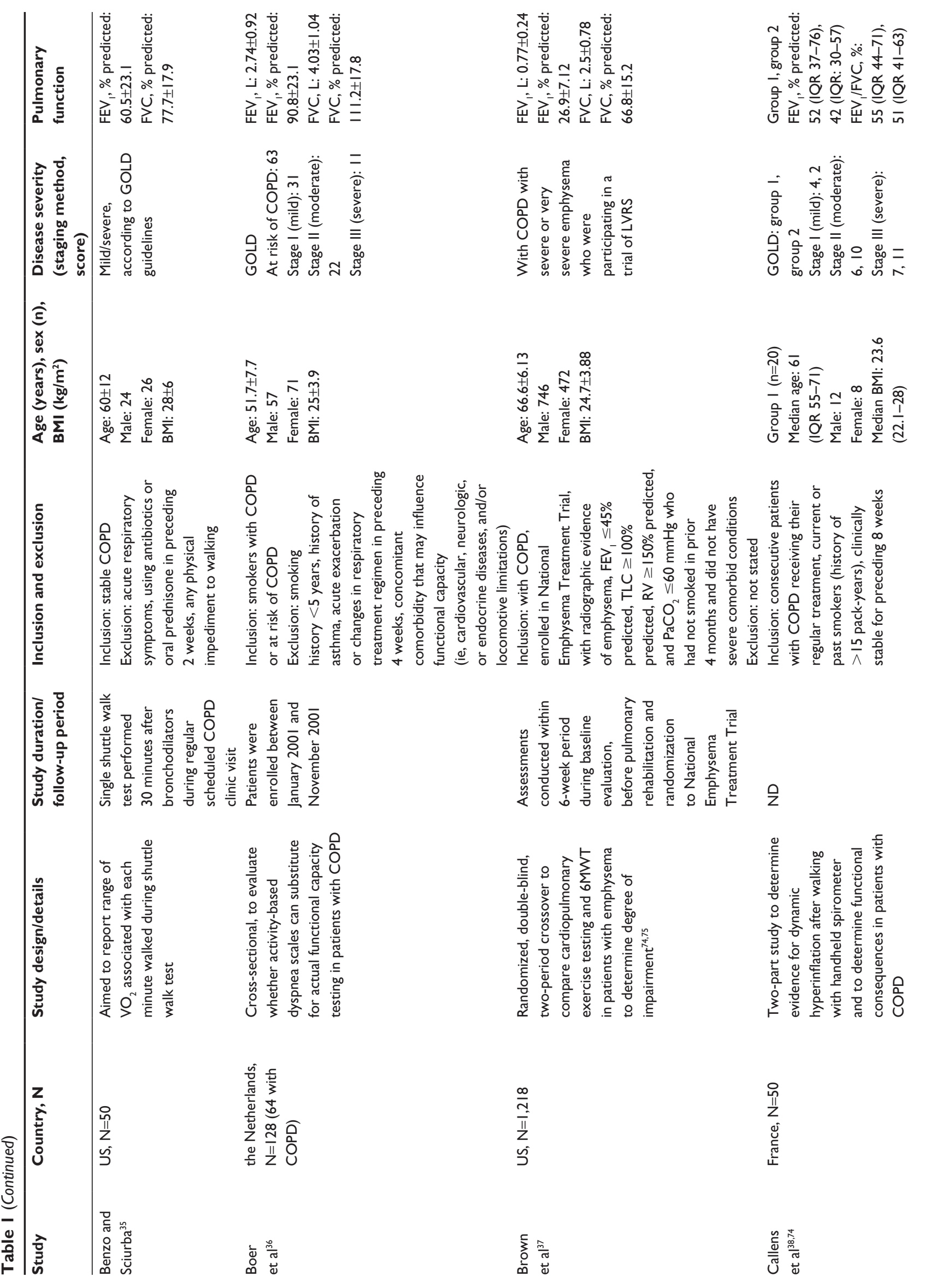




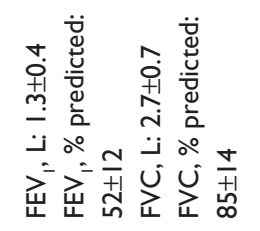

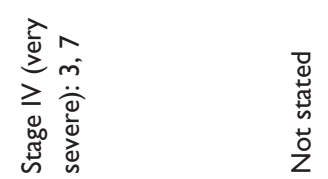

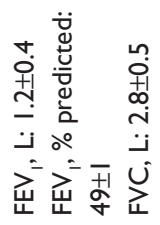

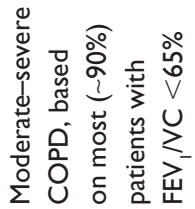

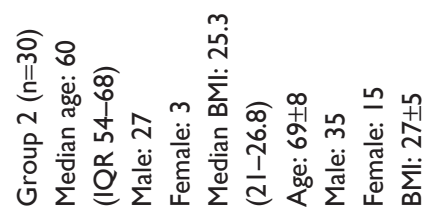

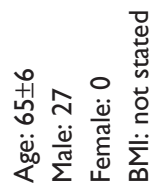
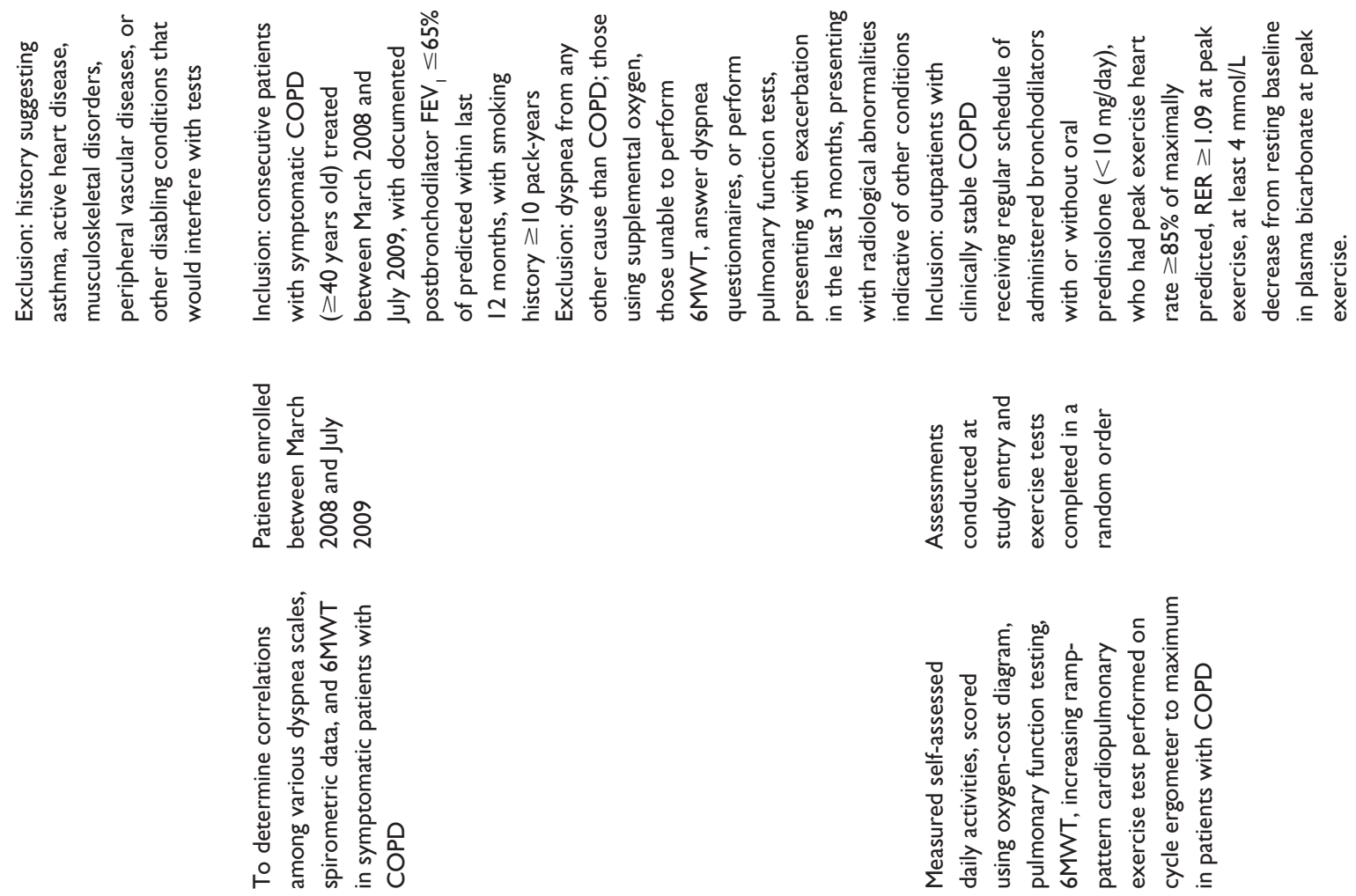

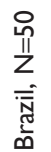

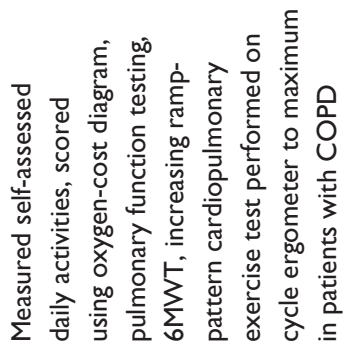

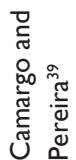

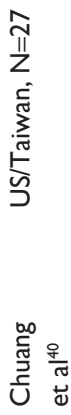




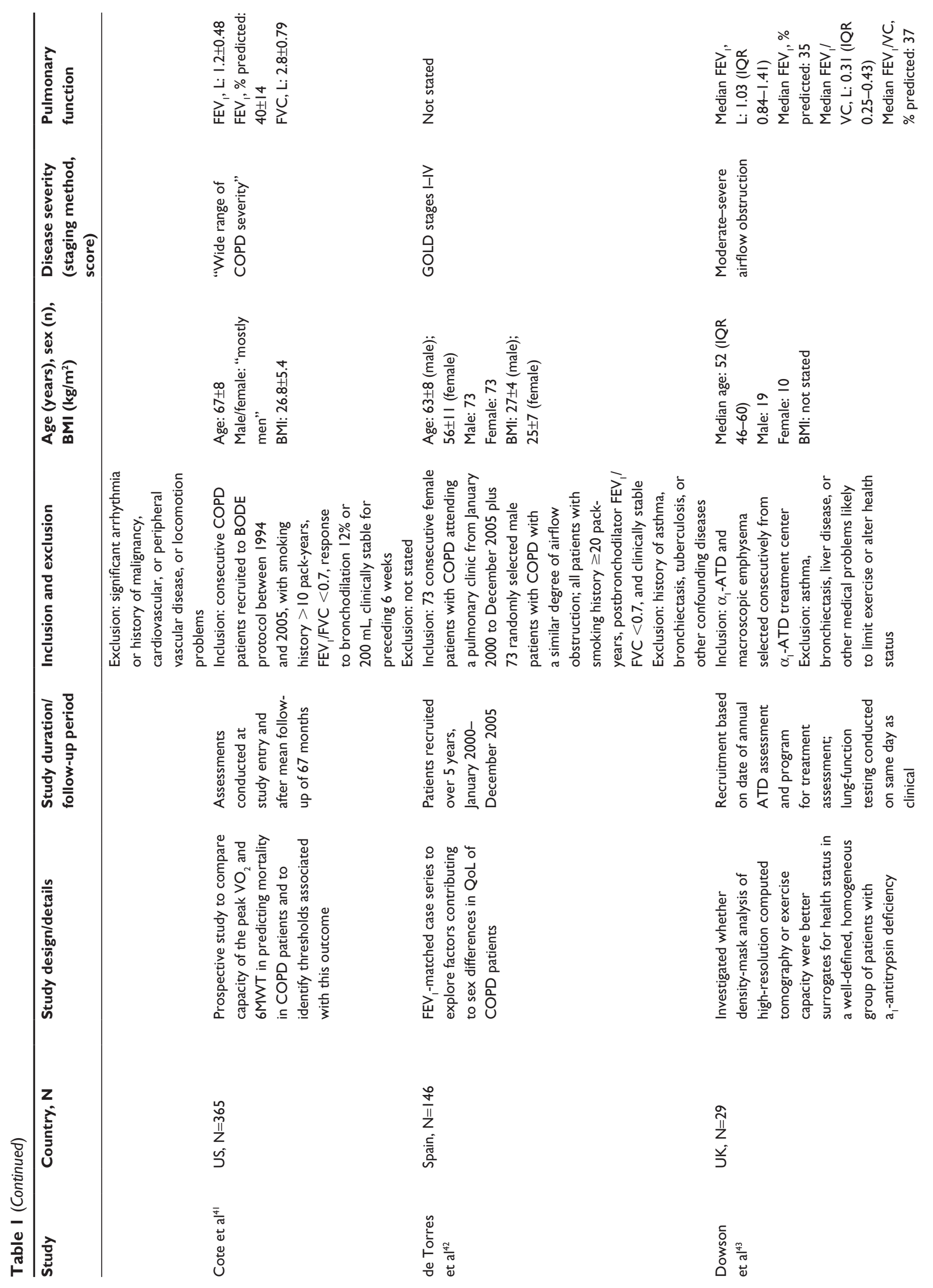



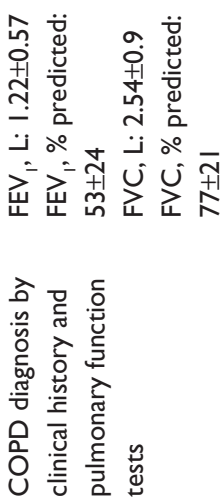

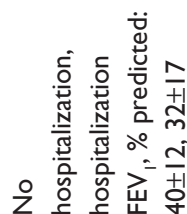

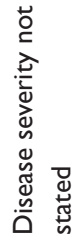

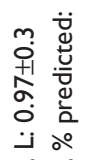

希昰市

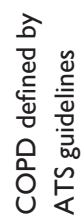

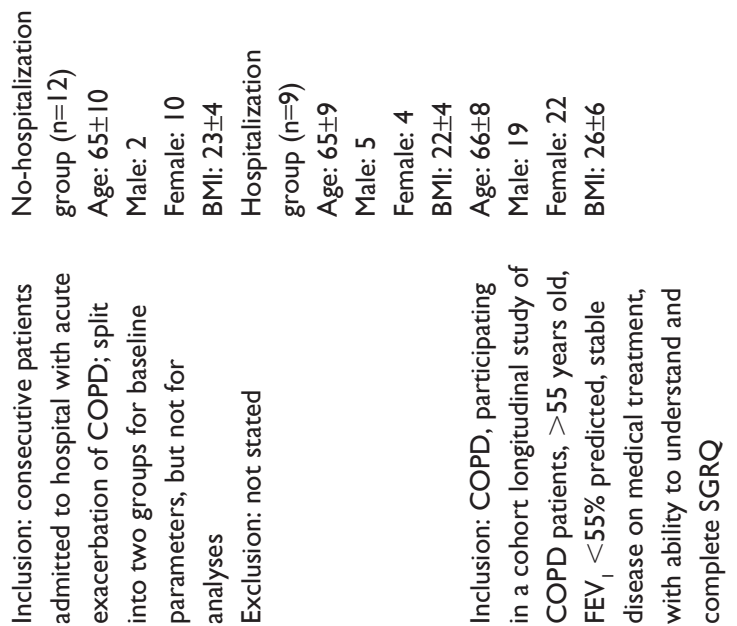
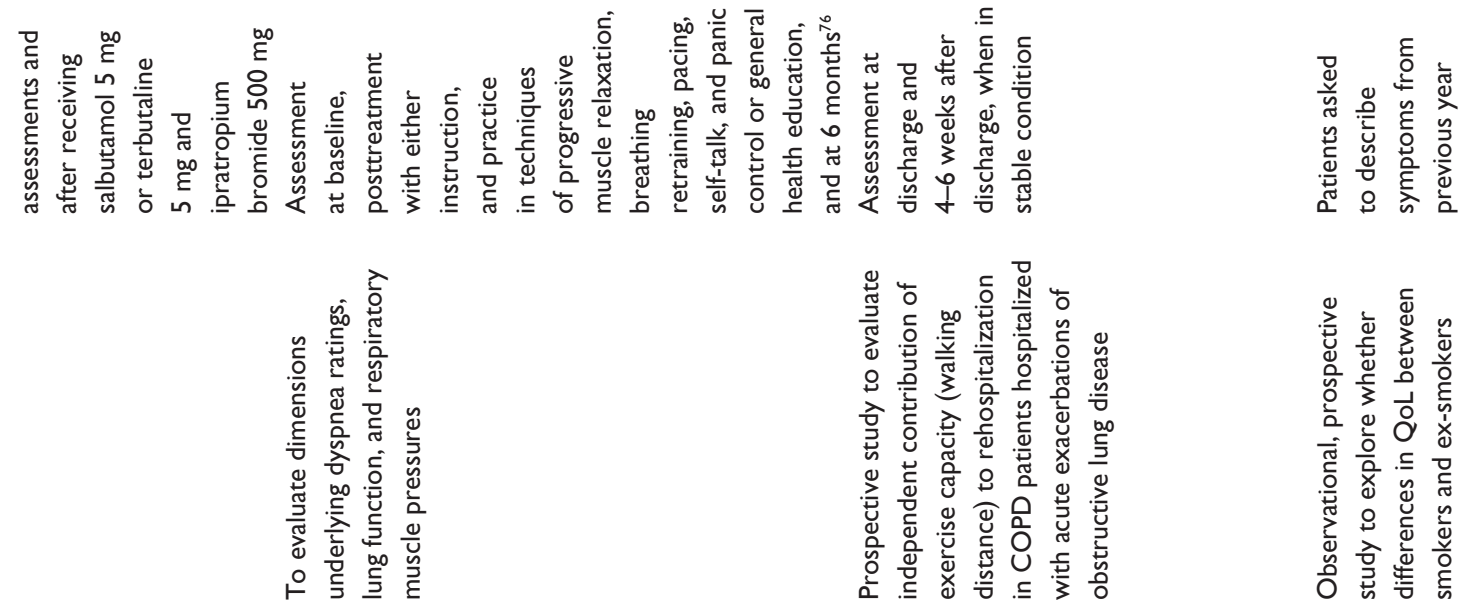

y
II
v
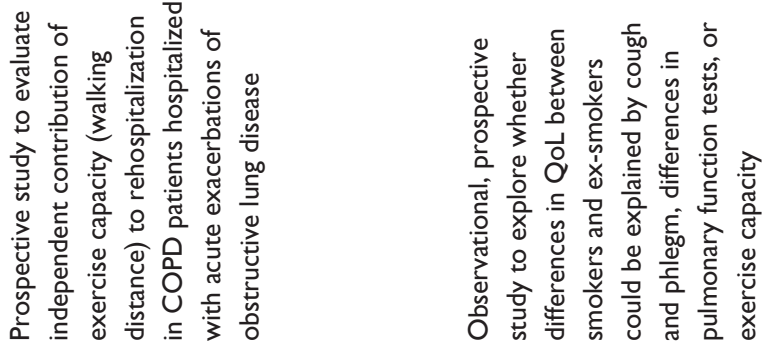

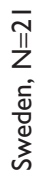

I
Z
ป

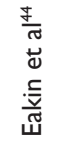

离先

$\frac{\sqrt{\frac{\pi}{2}}}{\frac{\pi}{\frac{\pi}{2}}}$ 


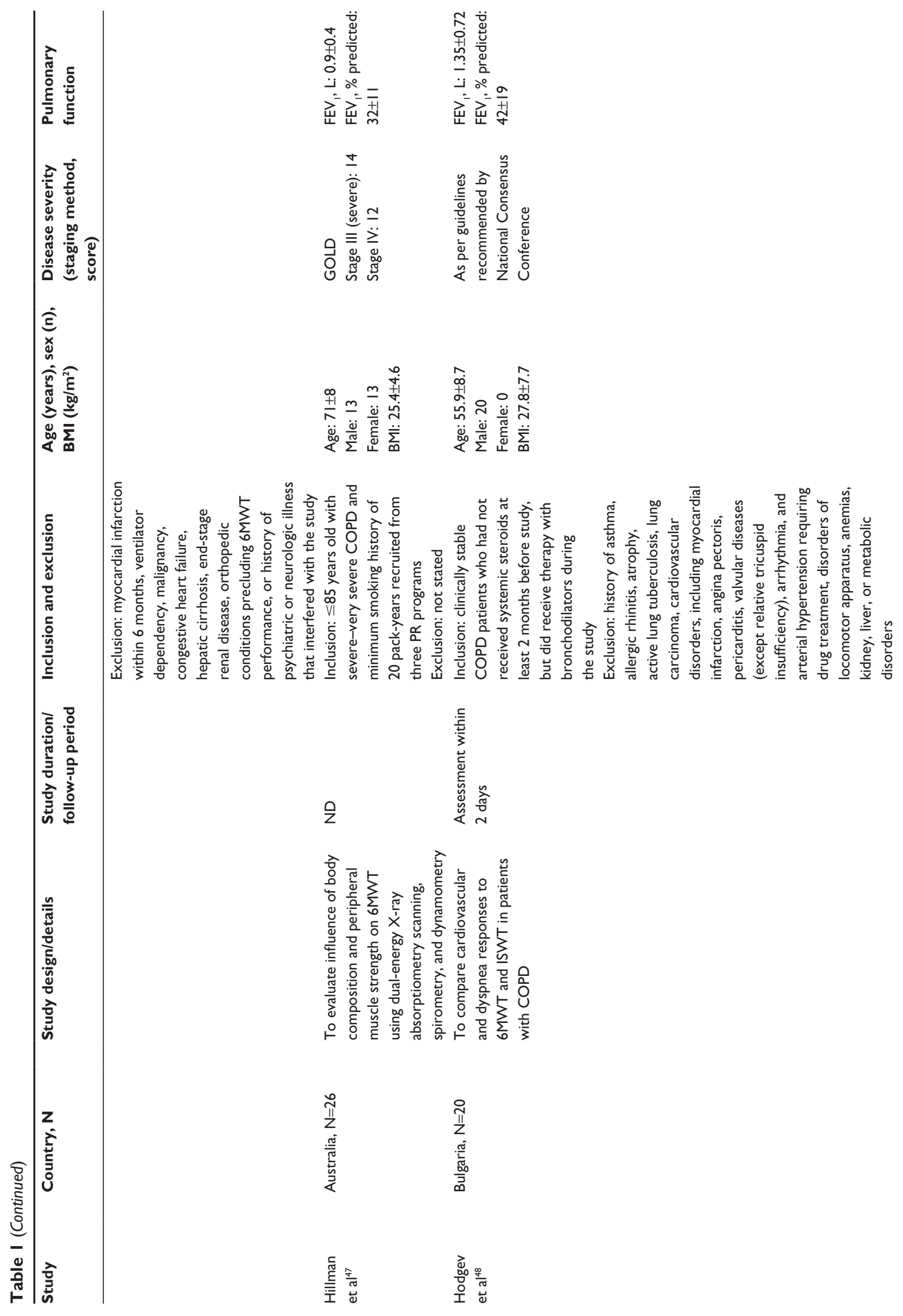




\section{กิ \\ 总 \\ ن \\ 窟它}

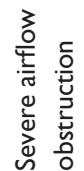

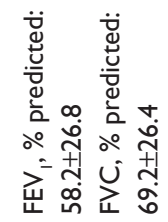

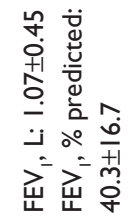

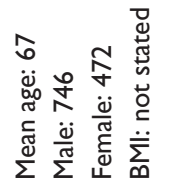

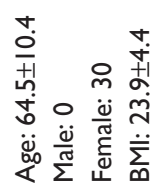

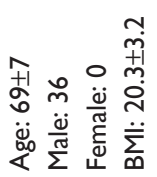
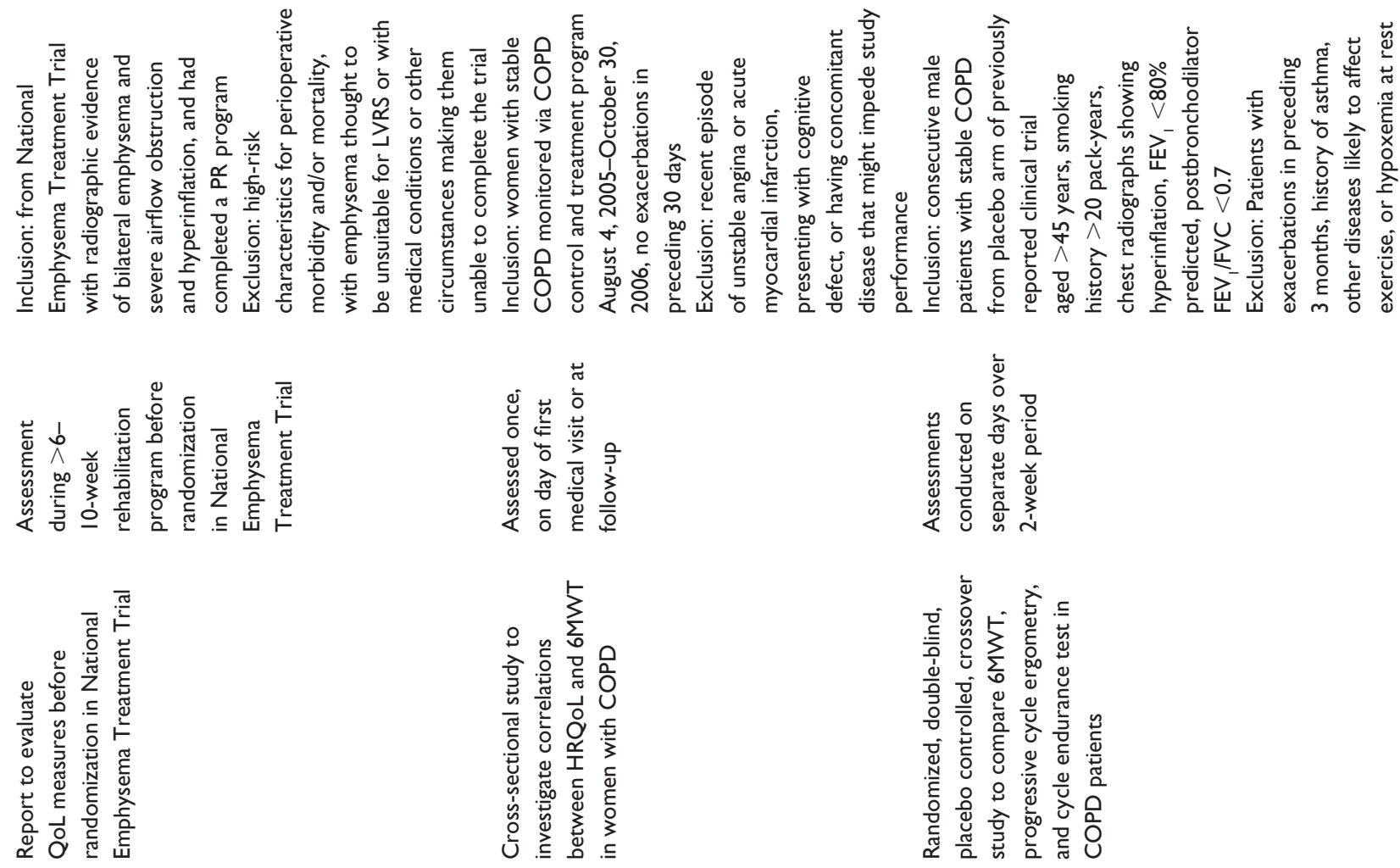

$\frac{\infty}{N}$
$\frac{11}{2}$
v

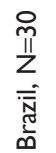

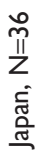

$\frac{\sqrt{\frac{\pi}{2}}}{\frac{\pi}{\frac{\pi}{0}}}$

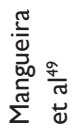

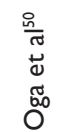




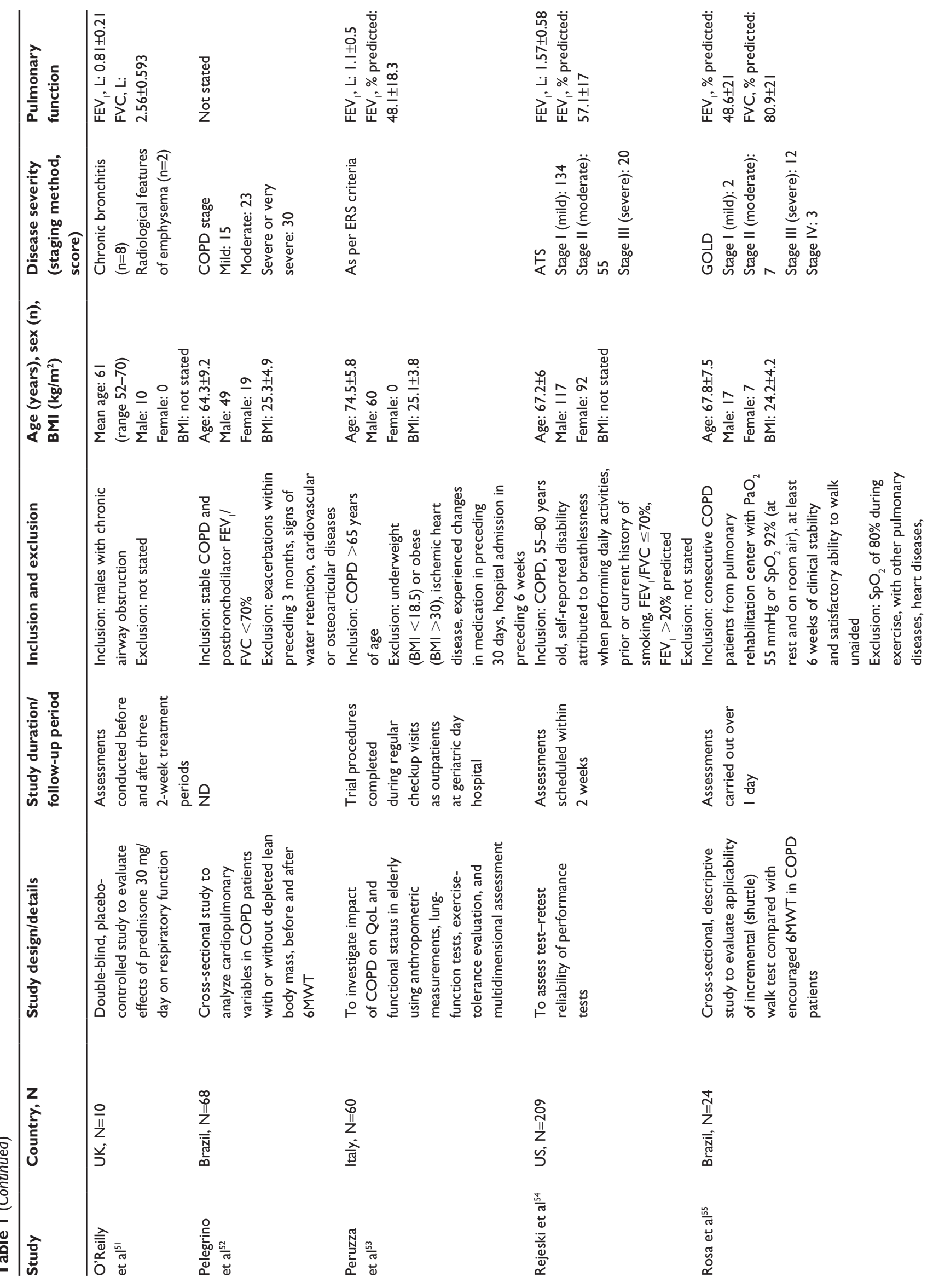




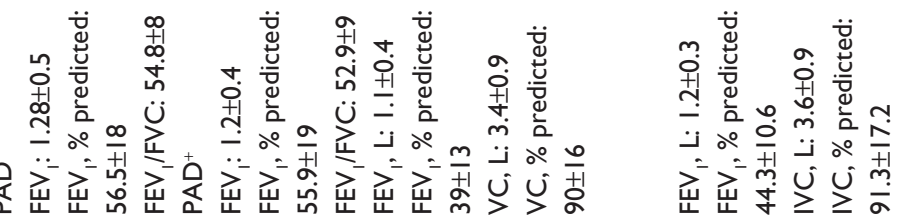

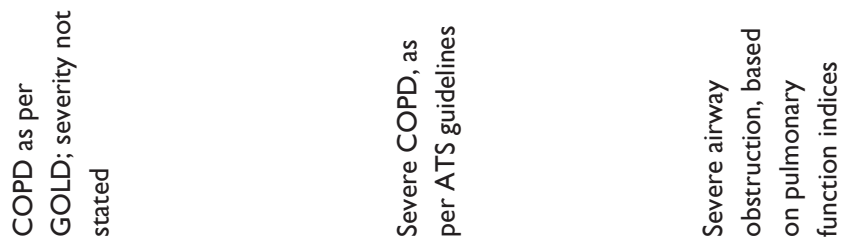

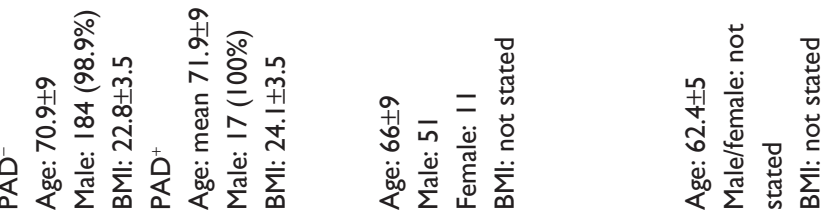

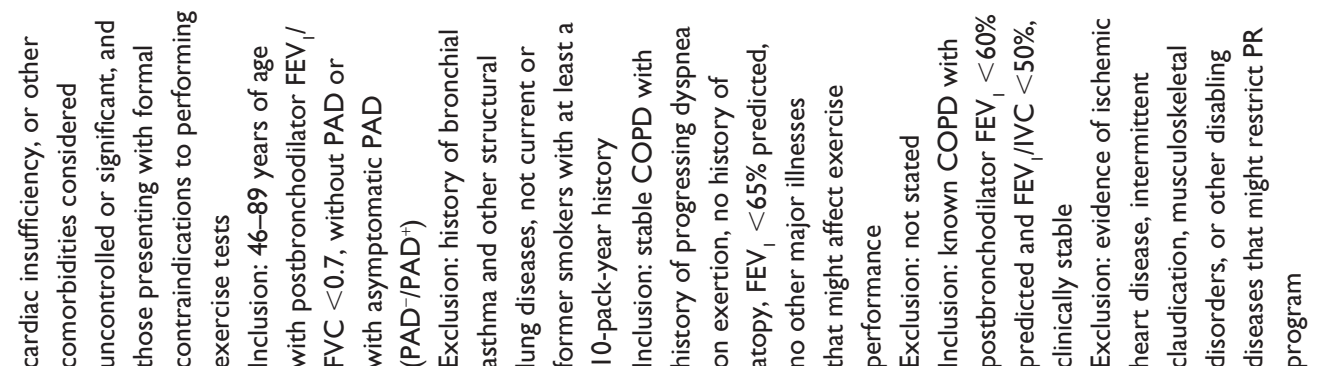

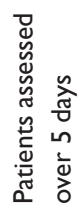

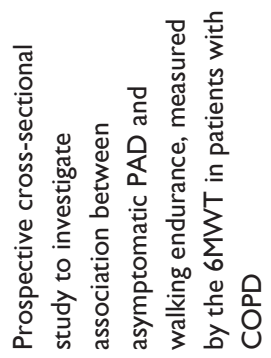

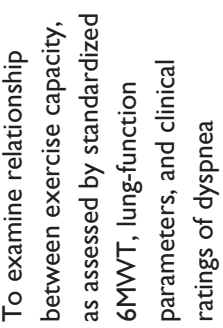

II

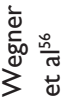
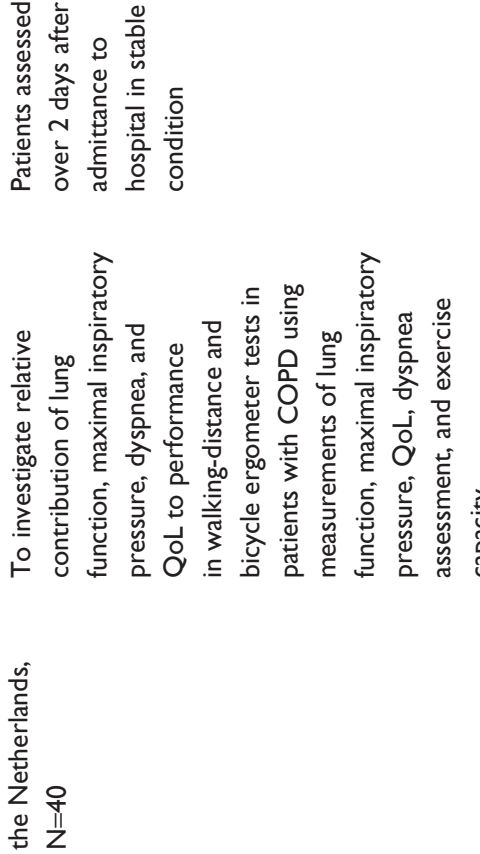

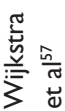

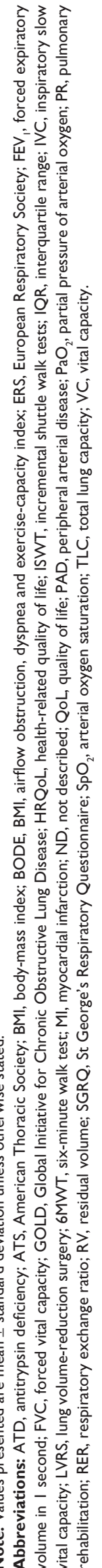


Table 2 Correlations between exercise test outcomes and selected quality-of-life PRO measures

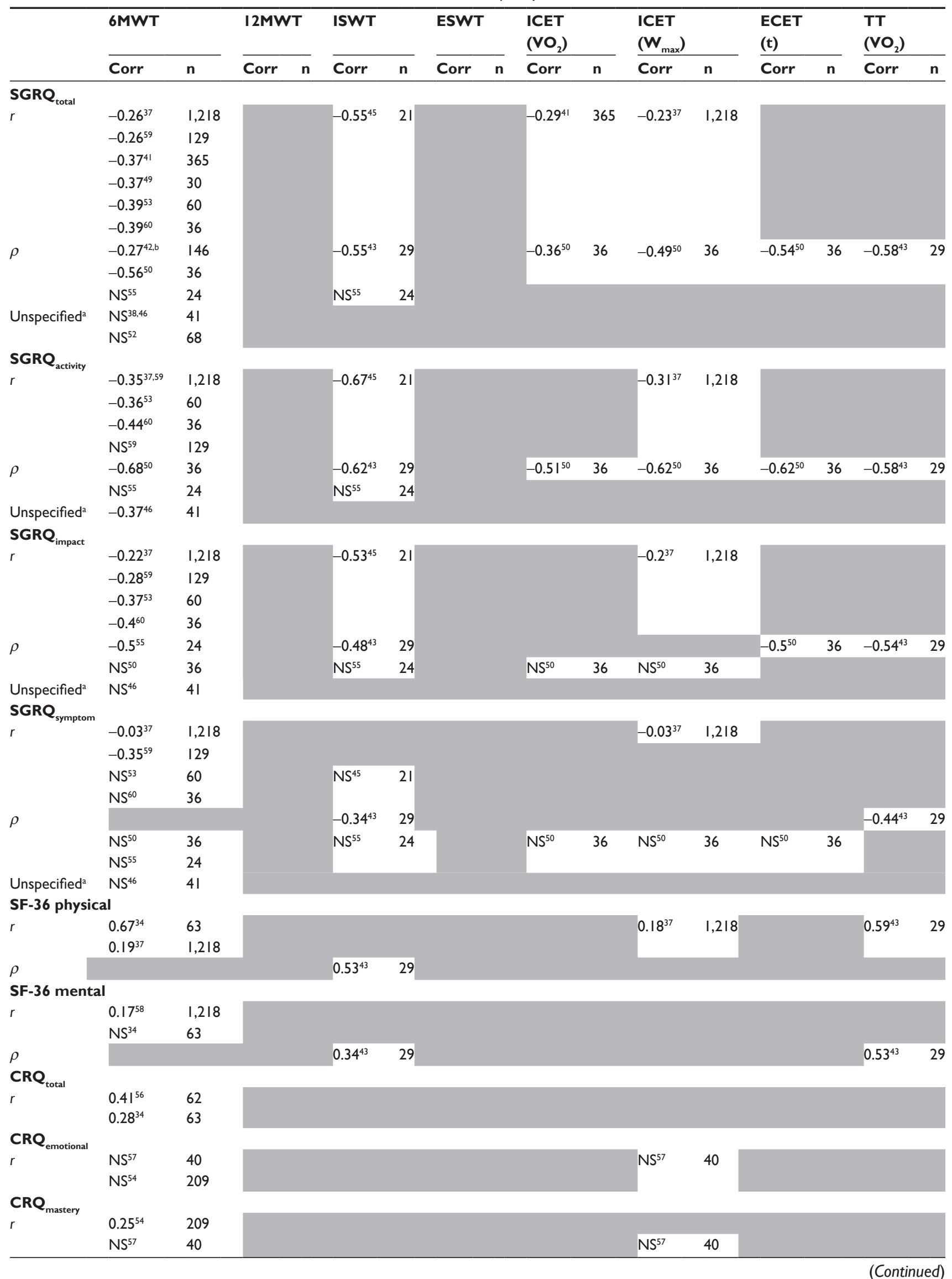


Table 2 (continued)

\begin{tabular}{|c|c|c|c|c|c|c|c|c|c|c|c|c|c|c|c|c|}
\hline & \multicolumn{2}{|c|}{ 6MWT } & \multicolumn{2}{|c|}{ I $2 \mathrm{MWT}$} & \multicolumn{2}{|c|}{ ISWT } & \multicolumn{2}{|c|}{ ESWT } & \multicolumn{2}{|c|}{$\begin{array}{l}\text { ICET } \\
\left(\mathrm{VO}_{2}\right)\end{array}$} & \multicolumn{2}{|c|}{$\begin{array}{l}\text { ICET } \\
\left(W_{\max }\right)\end{array}$} & \multicolumn{2}{|c|}{$\begin{array}{l}\text { ECET } \\
(\mathrm{t})\end{array}$} & \multicolumn{2}{|c|}{$\begin{array}{l}\text { TT } \\
\left(\mathrm{VO}_{2}\right)\end{array}$} \\
\hline & Corr & $\mathbf{n}$ & Corr & $\mathbf{n}$ & Corr & $\mathbf{n}$ & Corr & $\mathbf{n}$ & Corr & $\mathbf{n}$ & Corr & $\mathbf{n}$ & Corr & $\mathbf{n}$ & Corr & n \\
\hline \multicolumn{17}{|l|}{$\mathrm{CRQ}_{\text {fatigue }}$} \\
\hline \multirow[t]{2}{*}{$r$} & $0.25^{54}$ & 209 & & & & & & & & & & & & & & \\
\hline & $\mathrm{NS}^{57}$ & 40 & & & & & & & & & $N S^{57}$ & 40 & & & & \\
\hline
\end{tabular}

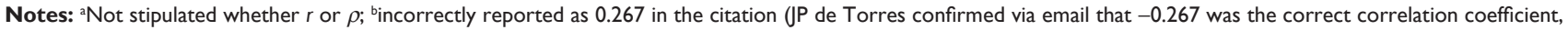
which has been reported to two decimal places here). Shaded areas indicate that no data were found for the relevant association.

Abbreviations: 6MWT, 6-minute walk test; I2MWT, 12-minute walk test; Corr, correlation; CRQ, Chronic Respiratory Disease Questionnaire; ECET, endurance cycle ergometer test; ESWT, endurance shuttle walk test; ICET, incremental cycle ergometer test; ISWT, incremental shuttle walk test; NS, no significance (reported); VO ${ }_{2}$, oxygen consumption; PRO, patient-reported outcome; SF-36, 36-item Short-Form Health Survey; SGRQ, St George's Respiratory Questionnaire; t, time; TT, treadmill test; W ${ }_{\text {max' }}$ highest workload achieved.

found a weak correlation between the $\mathrm{SGRQ}_{\text {impact }}$ and the $\operatorname{ICET~W}_{\max }(r=-0.20){ }^{37}$

\section{St George's Respiratory Questionnaire symptom domain}

Very limited data were found concerning associations between the SGRQ symptom domain score $\left(\mathrm{SGRQ}_{\text {symptom }}\right)$ and exercise test outcomes (Table 2). Five studies ${ }^{46,50,53,55,60}$ of seven $37,46,50,53,55,59,60$ assessing the association between

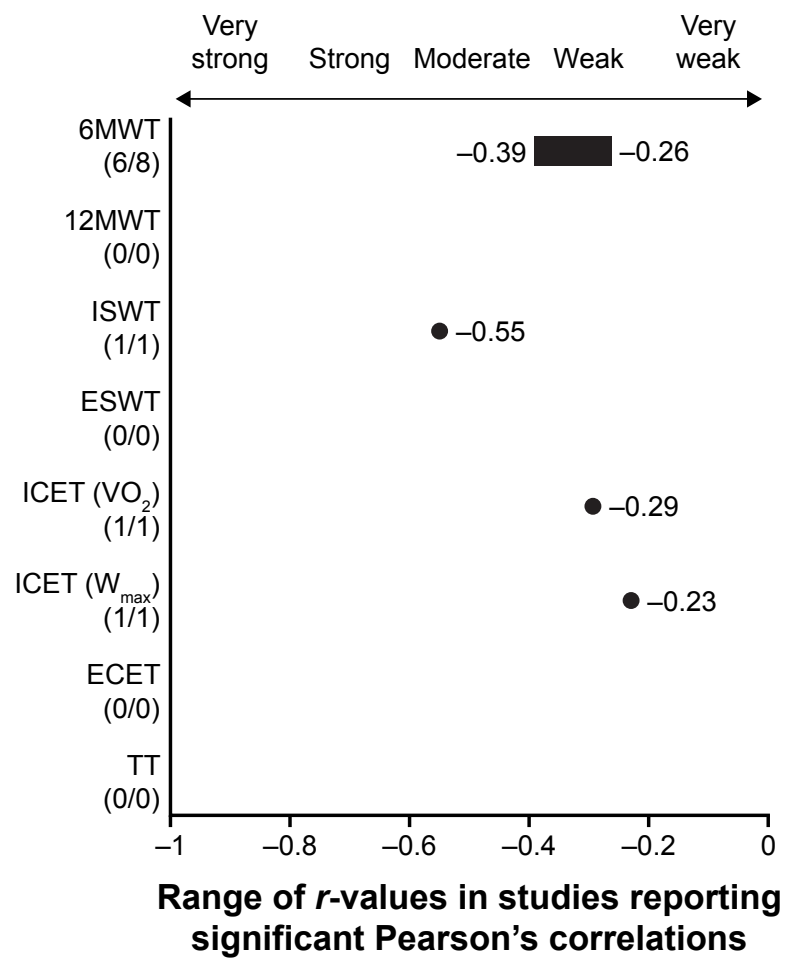

Figure 2 Pearson's correlations in studies reporting significant associations between exercise test outcomes and SGRQ ${ }_{\text {total }}$.

Notes: Numbers in parentheses refer to studies reporting significant correlations/ total number of studies reporting Pearson's correlations. Negative correlations indicate reduced $S G R Q_{\text {total }}$ scores with improvements in exercise test performance. Lower SGRQ scores indicate improved health status.

Abbreviations: 6MWT, 6-minute walk test; 12MWT, 12-minute walk test; ECET, endurance cycle ergometer test; ESWT, endurance shuttle walk test; ICET, incremental cycle ergometer test; ISWT, incremental shuttle walk test; peak $\mathrm{VO}_{2}$, peak rate of oxygen consumption; $\mathrm{SGRQ}_{\text {total }}$, St George's Respiratory Questionnaire total score; TT, treadmill test; $W_{\text {max }}$, highest workload achieved.
$\mathrm{SGRQ}_{\text {symptom }}$ and $6 \mathrm{MWT}$ distance reported nonsignificant relationships. In the two studies that reported significant correlations between $\mathrm{SGRQ}_{\text {symptom }}$ and $6 \mathrm{MWT}$, very weak negative $(-0.03)^{37}$ and weak negative $(-0.35)^{59}$ correlations were found. Two studies ${ }^{45,55}$ of three ${ }^{43,45,55}$ examining ISWT found no correlation with $\mathrm{SGRQ}_{\text {symptom }}$. One article reported weak $(\rho=-0.34)$ and moderate $(\rho=-0.44)$ correlations between the $\mathrm{SGRQ}_{\text {symptom }}$ and the ISWT and TT, respectively. ${ }^{43}$

\section{St George's Respiratory Questionnaire for COPD}

One study publication reported on the relationship between the 6MWT and the disease-specific SGRQ for COPD (SGRQ-c); however, this study examined risk factors, and reported only that worse (higher) SGRQ-c scores were an independent predictor of lower 6MWT distances. ${ }^{62}$

\section{Other quality-of-life instruments}

Too few publications reporting associations between exercise test outcomes and the SF-36, CRQ, or EQ-5D PROs were found to enable any meaningful assessment of their relationships.

\section{Correlations between exercise test outcomes and self-reported breathlessness}

Associations between the BDI and exercise test outcomes were reported for six studies (Table 3; Figure 3). ${ }^{36,44,48,52,55,56}$ Four articles noted an association between the 6MWT and BDI score: three publications reported moderate-very strong Pearson's correlations $(r=0.47-0.86),{ }^{44,48,56}$ with another reporting a moderate Spearman's correlation $(\rho=0.49) .{ }^{55}$ Only one study assessing this relationship found no significant correlation. ${ }^{52}$ Two additional publications reported moderate-strong Pearson's correlations between the BDI and ISWT $\left(-0.46^{36}\right.$ and $\left.0.76^{48}\right)$, with another reporting no significant correlation..$^{55}$ The positive correlations demonstrated in these studies show that PRO 
Table 3 Correlations between exercise test outcomes and selected patient-reported breathlessness measures

\begin{tabular}{|c|c|c|c|c|c|c|c|c|c|c|c|c|c|c|c|c|}
\hline & \multicolumn{2}{|l|}{ 6MWT } & \multicolumn{2}{|c|}{ I 2MWT } & \multicolumn{2}{|l|}{ ISWT } & \multicolumn{2}{|c|}{ ESWT } & \multicolumn{2}{|l|}{$\begin{array}{l}\text { ICET } \\
\left(\mathrm{VO}_{2}\right)\end{array}$} & \multicolumn{2}{|c|}{$\begin{array}{l}\text { ICET } \\
\left(W_{\max }\right) \\
\end{array}$} & \multicolumn{2}{|c|}{$\begin{array}{l}\text { ECET } \\
(t) \\
\end{array}$} & \multicolumn{2}{|c|}{$\begin{array}{l}\mathrm{TT} \\
\left(\mathrm{VO}_{2}\right)\end{array}$} \\
\hline & Corr & $\mathbf{n}$ & Corr & $\mathbf{n}$ & Corr & $\mathbf{n}$ & Corr & $\mathbf{n}$ & Corr & $\mathbf{n}$ & Corr & $\mathbf{n}$ & Corr & $\mathbf{n}$ & Corr & $\mathbf{n}$ \\
\hline \multicolumn{17}{|c|}{ BDI } \\
\hline \multirow[t]{4}{*}{$r$} & $(0.47,0.65)^{44}$ & 143 & & & $-0.46^{36}$ & 64 & & & & & & & & & & \\
\hline & $0.86^{48}$ & 20 & & & $0.76^{48}$ & 20 & & & & & & & & & & \\
\hline & $0.54^{56}$ & 62 & & & & & & & & & & & & & & \\
\hline & $\mathrm{NS}^{52}$ & 68 & & & & & & & & & & & & & & \\
\hline \multirow[t]{2}{*}{$\rho$} & $0.49^{55}$ & 24 & & & & & & & & & & & & & & \\
\hline & & & & & $\mathrm{NS}^{55}$ & 24 & & & & & & & & & & \\
\hline \multicolumn{17}{|c|}{ OCD } \\
\hline \multirow[t]{3}{*}{$r$} & $0.4^{40}$ & 27 & $0.496^{51}$ & 10 & $0.33^{36}$ & 64 & & & & & & & & & & \\
\hline & $(0.49,0.34)^{44}$ & 143 & & & & & & & & & & & & & & \\
\hline & $0.52^{56}$ & 62 & & & & & & & & & & & & & & \\
\hline \multirow[t]{2}{*}{$\rho$} & $0.66^{50}$ & 36 & & & & & & & $0.6 I^{50}$ & 36 & $0.74^{50}$ & 36 & $0.59^{50}$ & 36 & & \\
\hline & $\mathrm{NS}^{39}$ & 50 & & & & & & & & & & & & & & \\
\hline \multicolumn{17}{|c|}{$M R C / m M R C$} \\
\hline \multirow[t]{6}{*}{$r$} & $-0.667^{61}$ & 200 & & & $\begin{array}{l}\text { Correlated } \\
(r \text { not stated })^{35}\end{array}$ & 50 & & & $-0.38^{41}$ & 365 & 39 & 50 & & & & \\
\hline & $-0.51^{53}$ & 60 & & & & & & & & & & & & & & \\
\hline & $-0.52^{41}$ & 365 & & & & & & & & & & & & & & \\
\hline & $\begin{array}{l}-0.63^{56} \text { (reported as } \\
\text { negative in Table } 2 \text {, } \\
\text { but positive in text) }\end{array}$ & 62 & & & & & & & & & & & & & & \\
\hline & $-0.7^{47}$ & 26 & & & & & & & & & & & & & & \\
\hline & $\mathrm{NS}^{52}$ & 68 & & & & & & & & & & & & & & \\
\hline \multirow[t]{2}{*}{$\rho$} & $-0.5 I^{39}$ & 50 & & & & & & & & & & & & & & \\
\hline & $-0.39^{38}$ & 50 & & & & & & & & & & & & & & \\
\hline
\end{tabular}

Notes: Parenthesis enclose results of different subgroups within the same study; shaded areas indicate that no data were found for the relevant association.

Abbreviations: 6MWT, 6-minute walk test; I2MWT, I2-minute walk test; BDI, Baseline Dyspnea Index; Corr, correlation; ECET, endurance cycle ergometer test; ESWT, endurance shuttle walk test; ICET, incremental cycle ergometer test; ISWT, incremental shuttle walk test; mMRC, modified Medical Research Council (dyspnea scale); NS, no significance (reported); OCD, oxygen-cost diagram; $\mathrm{VO}_{2}$, oxygen consumption; $\mathrm{t}$, test; TT, treadmill test; $\mathrm{W}_{\text {max }}$, highest workload achieved.

scores increase as exercise performance improves, indicating improved health status.

Associations between OCD and exercise test outcomes were reported in seven articles (Table 3; Figure 3). ${ }^{36,39,40,44,50,51,56}$ Of these, three publications reported weak-moderate Pearson's correlations between the 6MWT and the OCD, ranging from 0.34 to $0.52,{ }^{40,44,56}$ with a further study finding a strong Spearman's correlation $(\rho=0.66) .{ }^{50}$ Only one study found no significant correlation between the 6MWT and the OCD ${ }^{39}$ Single articles noted weak-moderate correlations for the 12MWT $(r=0.50)^{51}$ and the ISWT $(r=0.33),{ }^{36}$ and a further paper reported strong Spearman's correlations of 0.61 and 0.74 for the ICET, when peak $\mathrm{VO}_{2}$ and $\mathrm{W}_{\max }$ were used for the main outcome measure, respectively. ${ }^{50}$ The same study also found moderate $(\rho=0.59)$ correlations between the OCD and the ECET. Of six studies ${ }^{41,47,52,53,56,61}$ reporting Pearson's correlations between the $6 \mathrm{MWT}$ and the $\mathrm{MRC} / \mathrm{mMRC}$ scale, five ${ }^{41,47,53,56,61}$ reported $r$-values ranging from -0.51 to -0.7 . However, one of these studies reporting Pearson's correlations between the 6MWT and the MRC/mMRC scale described $r^{2}$ rather than $r$. For the basis of this analysis, this study was included under the assumption that a Pearson's correlation was used and $r^{2}$ was a typographical error. ${ }^{61}$ One study $^{52}$ reported no significant correlation (Table 3; Figure 3), and another two studies reported a significant Spearman's correlation between MRC/mMRC and the 6MWT $\left(-0.51^{39}\right.$ and $\left.-0.39^{38}\right)$. The negative correlations demonstrated in these studies show that PRO scores decrease as exercise performance improves, indicating improved health status.

\section{Discussion}

This systematic review has shown that there are limited studies available reporting on the correlations between exercise test outcomes and PROs. Of these, the body of evidence describing a relationship between these outcome measures is even smaller. The most commonly reported association was between $\mathrm{SGRQ}_{\text {total }}$ outcomes and distance covered in the 6MWT. Though typically significant, these correlations were generally weak-moderate. The available evidence also showed correlations between the $S G R Q_{\text {total }}$ and $S G R Q_{\text {activity }}$ 


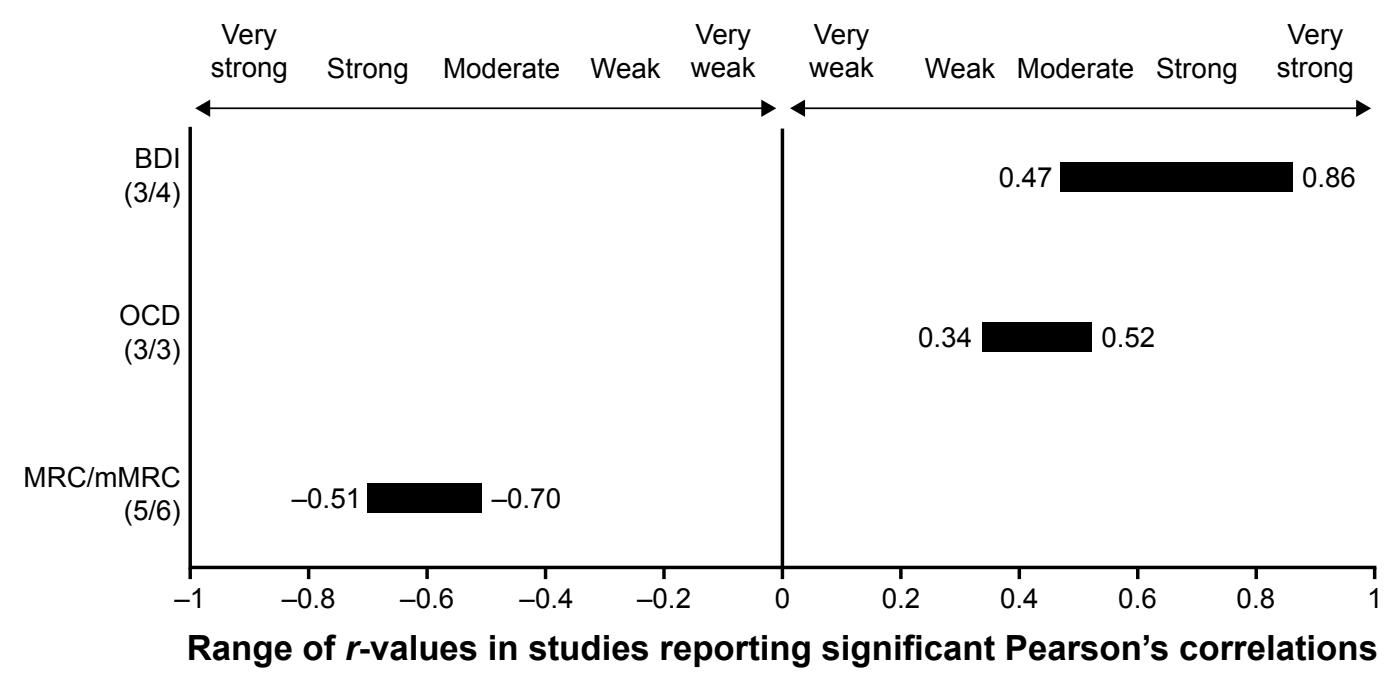

Figure 3 Pearson's correlations in studies reporting significant associations between the 6-minute walk-test outcomes and breathlessness PROs.

Notes: Numbers in parentheses refer to studies reporting significant correlations/total number of studies reporting Pearson's correlations. The two positive correlations shown here indicate higher PRO scores with improvements in exercise test performance. Higher PRO scores indicate improved health status. Negative correlations indicate lower PRO scores with improvements in exercise test performance. Lower PRO scores indicate improved health-related quality of life.

Abbreviations: BDI, Baseline Dyspnea Index; mMRC, modified Medical Research Council (dyspnea scale); PRO, patient-reported outcome; OCD, oxygen-cost diagram.

and the ISWT, ICET ( $\left.\mathrm{W}_{\max }\right)$, ECET, and TT, which tended to be moderate-strong. No studies were found that assessed associations between exercise test and SGRQ outcomes (including all subscales) for 12MWT and ESWT. However, all of these correlations must be considered in the context of substantial heterogeneity in study design, disease severity, and sample size.

The majority of studies investigating associations between exercise test outcomes and self-reported breathlessness scores, such as the BDI, OCD, and $\mathrm{mMRC}$, have used the 6MWT. The PROs of these tended to exhibit at least moderate correlations with exercise test outcomes. In particular, the BDI was generally reported to have a moderate-very strong Pearson's correlation with the 6MWT in three of four studies, with a further study reporting a moderate Spearman's correlation for this relationship.

Among included studies, there was a wide range of study designs and patient cohorts. As high-quality evidence is limited in this field, observational studies were combined with the results of randomized controlled trials, with the risk of affording similar weight to their interpretation. It is thus possible that significant associations could be underrecognized, owing to a type II statistical reporting error, a factor that must be considered when designing or interpreting studies to assess these exercise tests. Many of these studies reported nonsignificant correlations. Given the number of studies with small patient numbers that did report significant correlations, sample size is unlikely to be a factor in the nonsignificant correlations demonstrated by some studies. However, substantial heterogeneity was observed among some of the patient populations in terms of baseline demographics.

There are also inconsistencies in the way in which results were reported, with some study publications reporting incongruous negative/positive correlations compared with others describing the same relationship. It is unclear whether this reflects differences in the way in which the instruments were used or the way in which the statistical tests were applied between instruments and exercise test outcomes. We have presented such values as reported in the source articles. Additionally, the inclusion criteria and COPD severity are often not clearly stated in the articles included in this review. There is thus a risk that the patients in the studies included were not a broadly homogeneous group. Therefore, we would recommend that future studies clearly state inclusion criteria and the clinical rationale for diagnosis whenever possible.

Although not included in the review, consideration should also be given to the correlations demonstrated in studies that fell outside the selected search criteria. Several studies that were not included here showed significant relationships between exercise and breathlessness or PROs, either demonstrating correlations with new tools or using correlation coefficients other than Pearson's or Spearman's. For example, associations between exercise capacity and the i-BODE index (body-mass index, airflow obstruction, dyspnea, exercise-capacity index [exercise-capacity measured using ISWT]), ${ }^{63}$ the Functional Assessment of Chronic Illness Therapy - fatigue (FACIT-F) instrument, ${ }^{64}$ the London Chest Activity of Daily Living (LCADL) scale, ${ }^{65}$ 
and the McGill Pain Questionnaire (MPQ) ${ }^{66}$ were identified in patients with COPD. Moreover, correlations between the 6MWT and the COPD assessment test (CAT) ${ }^{67}$ and between the desaturation:distance ratio (DDR) and Borg scale ${ }^{68}$ have been identified. The criteria chosen for this review were based on well-established PROs, rather than those that have been more recently developed. However, many of these more novel tests are increasing in popularity, and as such, further investigation into their correlation with breathlessness or HRQoL PROs will be useful to determine their relevance in predicting prognosis in patients with COPD.

Despite the noted limitations, three further recommendations can be drawn directly from these findings. First, one of our included studies reported correlations between two PRO measures $\left(\mathrm{SGRQ}_{\text {total }}\right.$ and OCD) and two common outcome measures for the ICET: peak $\mathrm{VO}_{2}$ and $\mathrm{W}_{\max }$. In both, the PRO was more closely correlated with the ICET $\mathrm{W}_{\text {max }}$ than the ICET peak $\mathrm{VO}_{2}$. This finding complements that of another systematic review conducted by these authors, ${ }^{69}$ which showed that exercise test outcomes tended to be more closely correlated with $\mathrm{W}_{\max }$ as a measure of lung function than with peak $\mathrm{VO}_{2}$. It thus seems prudent to suggest that when investigating ICET, researchers report $\mathrm{W}_{\max }$ values as a priority.

Second, as a combination of the SF-36 and EQ-5D is the instrument of choice for assessing HRQoL of several health technology-assessment bodies, the lack of studies reporting associations between this outcome measure and exercise tests is of note. Clinical trial designers might usefully consider this in assessing the most appropriate clinical trial end point. Third, the results of this review suggest that exercise tests are not interchangeable, and as such, comparisons of different exercise tests in response to interventions are inappropriate.

In conclusion, these findings indicate that only limited evidence is available to support an association between exercise test outcomes and HRQoL and breathlessness PROs in patients with COPD. The evidence that does exist suggests a very weak-moderate negative correlation between the 6MWT and the SGRQ. Both moderate-strong positive and negative correlations between 6MWT outcomes and breathlessness were observed. It has not been possible to assess other tests adequately, though the limited data available suggest that the ISWT, ICET, ECET, and TT may be more closely associated with the SGRQ (and HRQoL outcomes) than the 6MWT. Recent guidelines on the diagnosis and treatment of COPD indicate that the assessment of disease severity is improved by using functional criteria, such as exercise capacity. ${ }^{4,70,71}$ However, the current evidence suggests that no single exercise test accurately reflects HRQoL or breathlessness in patients with COPD. Therefore, despite the paucity of data for some tests, it may be justified to conclude that these tests assess features not measured by these HRQoL outcomes. Consequently, a composite measurement assessing several factors reflective of COPD, such as the BODE index, which evaluates a surrogate of nutritional state (body-mass index), airflow obstruction $\left(\mathrm{FEV}_{1}\right)$, dyspnea (mMRC), and exercise capacity (6MWT), may be a more accurate measure of COPD severity and prognosis. ${ }^{72}$ The BODE index predicts the requirement for hospitalizations among patients with COPD better than either $\mathrm{FEV}_{1}$ or classic GOLD staging. ${ }^{73}$ It thus seems reasonable to surmise that individual PROs may have limited prognostic ability in patients with COPD, and should be supported by additional measurements wherever possible.

\section{Acknowledgments}

The authors would like to thank Martin Bell, Iain Fotheringham, and Sarah Cockle for their contribution to the original study, and Jelle Spoorendonk, Weiwei Xu, and Janita Balradi (Pharmerit International) for conducting the updated systematic literature review. Editorial support (in the form of writing assistance, assembling tables and figures, collating author comments, grammatical editing, and referencing) was provided by Rachael Baylie, $\mathrm{PhD}$ at Fishawack Indicia Ltd, UK, and was funded by GSK. This study was funded by GSK.

\section{Author contributions}

All authors contributed to the conception and design of the study, analysis and interpretation of data, and revision of the manuscript, and approved the final version of the manuscript.

\section{Disclosure}

YSP, JHR, EL, and MD are current employees of GlaxoSmithKline and hold stocks in GlaxoSmithKline. SJS was involved with the development of the incremental shuttle walk test, and has served on advisory boards for GlaxoSmithKline. SJS was part funded by the National Institute for Health Research (NIHR) Collaboration for Leadership in Applied Health Research and Care East Midlands (CLAHRC EM). Support was also provided by the NIHR Leicester Respiratory Biomedical Research Unit. The views expressed are those of the authors, and not necessarily those of the National Health Service (NHS), the NIHR, or the Department of Health. The work presented here, including 
the conduct of the study, data analysis, and interpretation, was funded by GSK (HO-12-12583).

\section{References}

1. Lopez AD, Shibuya K, Rao C, et al. Chronic obstructive pulmonary disease: current burden and future projections. Eur Respir J. 2006;27(2): 397-412.

2. Halbert RJ, Natoli JL, Gano A, Badamgarav E, Buist AS, Mannino DM. Global burden of COPD: systematic review and meta-analysis. Eur Respir J. 2006;28(3):523-532.

3. Mathers CD, Loncar D. Projections of global mortality and burden of disease from 2002 to 2030. PLoS Med. 2006;3(11):e442.

4. Global Initiative for Chronic Obstructive Lung Disease (GOLD). Global Strategy for the Diagnosis, Management, and Prevention of COPD. Bethesda (MD): GOLD; 2014.

5. Gosker HR, Lencer NH, Franssen FM, Van Der Vusse GJ, Wouters EF, Schols AM. Striking similarities in systemic factors contributing to decreased exercise capacity in patients with severe chronic heart failure or COPD. Chest. 2003;123(5):1416-1424.

6. O’Donnell DE, Gebke KB. Activity restriction in mild COPD: a challenging clinical problem. Int J Chron Obstruct Pulmon Dis. 2014;9:577-588.

7. Jones P, Miravitlles M, van der Molen T, Kulich K. Beyond FEV in COPD: a review of patient-reported outcomes and their measurement. Int J Chron Obstruct Pulmon Dis. 2012;7:697-709.

8. Cooper CB. The connection between chronic obstructive pulmonary disease symptoms and hyperinflation and its impact on exercise and function. Am J Med. 2006;119(10 Suppl 1):21-31.

9. Jones PW. Issues concerning health-related quality of life in COPD. Chest. 1995;107(5 Suppl):187S-193S.

10. Mahler DA, Harver A. A factor analysis of dyspnea ratings, respiratory muscle strength, and lung function in patients with chronic obstructive pulmonary disease. Am Rev Respir Dis. 1992;145(2 Pt 1):467-470.

11. Butland RJ, Pang J, Gross ER, Woodcock AA, Geddes DM. Two-, six-, and 12-minute walking tests in respiratory disease. Br Med J (Clin Res Ed). 1982;284(6329):1607-1608.

12. McGavin CR, Gupta SP, McHardy GJ. Twelve minute walking test for assessing disability in chronic bronchitis. Br Med J. 1976;1(6013): 822-823.

13. Singh SJ, Morgan MD, Scott S, Walters D, Hardman AE. Development of a shuttle walking test of disability in patients with chronic airways obstruction. Thorax. 1992;47(12):1019-1024.

14. Revill SM, Morgan MD, Singh SJ, Williams J, Hardman AE. The endurance shuttle walk: a new field test for the assessment of endurance capacity in chronic obstructive pulmonary disease. Thorax. 1999;54(3): 213-222.

15. Lu Y, Nyunt MS, Gwee X, et al. Life event stress and chronic obstructive pulmonary disease (COPD): associations with menta well-being and quality of life in a population-based study. BMJ Open. 2012;2(6):e001674.

16. Garrod R, Marshall J, Barley E, Jones PW. Predictors of success and failure in pulmonary rehabilitation. Eur Respir J. 2006;27(4):788-794.

17. Jones PW, Quirk FH, Baveystock CM, Littlejohns P. A self-complete measure of health status for chronic airflow limitation: the St George's Respiratory Questionnaire. Am Rev Respir Dis. 1992;145(6):1321-1327.

18. Wijkstra PJ, Van Altena R, Kraan J, Otten V, Postma DS, Koeter GH. Quality of life in patients with chronic obstructive pulmonary disease improves after rehabilitation at home. Eur Respir J. 1994;7(2): 269-273.

19. Fotheringham I, Meakin G, Punekar YS, Riley JH, Cockle SM, Singh SJ. Comparison of laboratory-and field-based exercise tests for COPD: a systematic review. Int J Chron Obstruct Pulmon Dis. 2015; 10:625-643.

20. Moher D, Liberati A, Tetzlaff J, Altman DG, Group P. Preferred reporting items for systematic reviews and meta-analyses: the PRISMA statement. BMJ. 2009;339:b2535.
21. Jenkinson C, Coulter A, Wright L. Short form 36 (SF36) health survey questionnaire: normative data for adults of working age. BMJ. 1993;306(6890):1437-1440.

22. EuroQol Group. EuroQol: a new facility for the measurement of healthrelated quality of life. Health Policy. 1990;16(3):199-208.

23. Brooks R. EuroQol: the current state of play. Health Policy. 1996; 37(1):53-72.

24. Guyatt GH, Berman LB, Townsend M, Pugsley SO, Chambers LW. A measure of quality of life for clinical trials in chronic lung disease. Thorax. 1987;42(10):773-778.

25. Mahler DA, Weinberg DH, Wells CK, Feinstein AR. The measurement of dyspnea: contents, interobserver agreement, and physiologic correlates of two new clinical indexes. Chest. 1984;85(6):751-758.

26. McGavin CR, Artvinli M, Naoe H, McHardy GJ. Dyspnoea, disability, and distance walked: comparison of estimates of exercise performance in respiratory disease. $\mathrm{Br}$ Med J. 1978;2(6132):241-243.

27. Fletcher CM, Elmes PC, Fairbairn AS, Wood CH. The significance of respiratory symptoms and the diagnosis of chronic bronchitis in a working population. $\mathrm{Br}$ Med J. 1959;2(5147):257-266.

28. Mahler DA, Rosiello RA, Harver A, Lentine T, McGovern JF, Daubenspeck JA. Comparison of clinical dyspnea ratings and psychophysical measurements of respiratory sensation in obstructive airway disease. Am Rev Respir Dis. 1987;135(6):1229-1233.

29. Jones PW, Quirk FH, Baveystock CM, Littlejohns P. A self-complete measure of health status for chronic airflow limitation: the St. George's Respiratory Questionnaire. Am Rev Respir Dis. 1992;145(6): 1321-1327.

30. Bestall J, Paul E, Garrod R, Garnham R, Jones P, Wedzicha J. Usefulness of the Medical Research Council (MRC) dyspnoea scale as a measure of disability in patients with chronic obstructive pulmonary disease. Thorax. 1999;54(7):581-586.

31. Global Initiative for Chronic Obstructive Lung Disease (GOLD). Global Strategy for the Diagnosis, Management, and Prevention of COPD. Bethesda (MD): GOLD; 2016.

32. Jenkinson C, Coulter A, Wright L. Short form 36 (SF36) health survey questionnaire: normative data for adults of working age. BMJ. 1993; 306(6890):1437-1440.

33. BMJ Publishing Group. Correlation and regression. Available from: http://www.bmj.com/about-bmj/resources-readers/publications/ statistics-square-one/11-correlation-and-regression. Accessed May 16, 2017.

34. Belza B, Steele BG, Hunziker J, Lakshminaryan S, Holt L, Buchner DM. Correlates of physical activity in chronic obstructive pulmonary disease. Nurse Res. 2001;50(4):195-202.

35. Benzo RP, Sciurba FC. Oxygen consumption, shuttle walking test and the evaluation of lung resection. Respiration. 2010;80(1):19-23.

36. Boer LM, Asijee GM, van Schayck OC, Schermer TR. How do dyspnoea scales compare with measurement of functional capacity in patients with COPD and at risk of COPD? Prim Care Respir J. 2012;21(2): 202-207.

37. Brown CD, Benditt JO, Sciurba FC, et al. Exercise testing in severe emphysema: association with quality of life and lung function. COPD. 2008;5(2):117-124.

38. Callens E, Graba S, Gillet-Juvin K, et al. Measurement of dynamic hyperinflation after a 6-minute walk test in patients with COPD. Chest. 2009;136(6):1466-1472.

39. Camargo LA, Pereira CA. Dyspnea in COPD: beyond the modified Medical Research Council scale. J Bras Pneumol. 2010;36(5):571-578.

40. Chuang ML, Lin IF, Wasserman K. The body weight-walking distance product as related to lung function, anaerobic threshold and peak $\mathrm{VO}_{2}$ in COPD patients. Respir Med. 2001;95(7):618-626.

41. Cote CG, Pinto-Plata V, Kasprzyk K, Dordelly LJ, Celli BR. The 6-min walk distance, peak oxygen uptake, and mortality in COPD. Chest. 2007;132(6):1778-1785.

42. de Torres JP, Casanova C, Hernandez C, et al. Gender associated differences in determinants of quality of life in patients with COPD: a case series study. Health Qual Life Outcomes. 2006;4:72. 
43. Dowson LJ, Newall C, Guest PJ, Hill SL, Stockley RA. Exercise capacity predicts health status in alpha1-antitrypsin deficiency. Am J Respir Crit Care Med. 2001;163(4):936-941.

44. Eakin EG, Kaplan RM, Ries AL, Sassi-Dambron DE. Patients' selfreports of dyspnea: An important and independent outcome in chronic obstructive pulmonary disease. Ann Behav Med. 1996;18(2):87-90.

45. Emtner MI, Arnardottir HR, Hallin R, Lindberg E, Janson C. Walking distance is a predictor of exacerbations in patients with chronic obstructive pulmonary disease. Respir Med. 2007;101(5):1037-1040.

46. Heijdra YF, Pinto-Plata VM, Kenney LA, Rassulo J, Celli BR. Cough and phlegm are important predictors of health status in smokers without COPD. Chest. 2002;121(5):1427-1433.

47. Hillman CM, Heinecke EL, Hii JW, Cecins NM, Jenkins SC, Eastwood PR. Relationship between body composition, peripheral muscle strength and functional exercise capacity in patients with severe chronic obstructive pulmonary disease. Intern Med J. 2012;42(5):578-581.

48. Hodgev VA, Aliman OI, Marinov BI, Kostianev SS, Mandulova PV. Cardiovascular and dyspnea response to six-minute and shuttle walk tests in COPD patients. Folia Med (Plovdiv). 2003;45(3):26-33.

49. Mangueira NM, Viega IL, Mangueira MA, Pinheiro AN, Costa MR. Correlation between clinical parameters and health-related quality of life in women with COPD. J Bras Pneumol. 2009;35(3):248-255.

50. Oga $T$, Nishimura $K$, Tsukino $M$, Hajiro T, Ikeda A, Mishima M. Relationship between different indices of exercise capacity and clinical measures in patients with chronic obstructive pulmonary disease. Heart Lung. 2002;31(5):374-381.

51. O'Reilly JF, Shaylor JM, Fromings KM, Harrison BD. The use of the 12 minute walking test in assessing the effect of oral steroid therapy in patients with chronic airways obstruction. $\mathrm{Br} J$ Dis Chest. 1982;76(4):374-382.

52. Pelegrino NR, Lucheta PA, Sanchez FF, Faganello MM, Ferrari R, de Godoy I. Influence of lean body mass on cardiopulmonary repercussions during the six-minute walk test in patients with COPD. $J$ Bras Pneumol. 2009;35(1):20-26.

53. Peruzza S, Sergi G, Vianello A, et al. Chronic obstructive pulmonary disease (COPD) in elderly subjects: impact on functional status and quality of life. Respir Med. 2003;97(6):612-617.

54. Rejeski WJ, Foley KO, Woodard CM, Zaccaro DJ, Berry MJ. Evaluating and understanding performance testing in COPD patients. J Cardiopulm Rehabil. 2000;20(2):79-88.

55. Rosa FW, Camelier A, Mayer A, Jardim JR. Evaluating physical capacity in patients with chronic obstructive pulmonary disease: comparing the shuttle walk test with the encouraged 6-minute walk test. $J$ Bras Pneumol. 2006;32(2):106-113.

56. Wegner RE, Jorres RA, Kirsten DK, Magnussen H. Factor analysis of exercise capacity, dyspnoea ratings and lung function in patients with severe COPD. Eur Respir J. 1994;7(4):725-729.

57. Wijkstra PJ, TenVergert EM, van der Mark TW, et al. Relation of lung function, maximal inspiratory pressure, dyspnoea, and quality of life with exercise capacity in patients with chronic obstructive pulmonary disease. Thorax. 1994;49(5):468-472.

58. Kaplan RM, Ries AL, Reilly J, Mohsenifar Z. Measurement of healthrelated quality of life in the national emphysema treatment trial. Chest. 2004;126(3):781-789.

59. Agrawal SR, Joshi R, Jain A. Correlation of severity of chronic obstructive pulmonary disease with health-related quality of life and six-minute walk test in a rural hospital of central India. Lung India. 2015;32(3):233-240.

International Journal of COPD

\section{Publish your work in this journal}

The International Journal of COPD is an international, peer-reviewed journal of therapeutics and pharmacology focusing on concise rapid reporting of clinical studies and reviews in COPD. Special focus is given to the pathophysiological processes underlying the disease, intervention programs, patient focused education, and self management protocols.
60. Bavarsad MB, Shariati A, Eidani E, Latifi M. Relationship between exercise capacity and clinical measures in patients with chronic obstructive pulmonary disease. Biomed Pharmacol J. 2015;8:1-7.

61. Sun KS, Lin MS, Chen YJ, Chen YY, Chen SC, Chen W. Is asymptomatic peripheral arterial disease associated with walking endurance in patients with COPD? Int J Chron Obstruct Pulmon Dis. 2015;10: 1487-1492.

62. Spruit MA, Watkins ML, Edwards LD, et al. Determinants of poor 6-min walking distance in patients with COPD: the ECLIPSE cohort. Respir Med. 2010;104(6):849-857.

63. Utku ES, Ergun PS, Kaymaz DY, et al. i-BODE for evaluating the efficiency of pulmonary rehabilitation in patients with COPD. Eur Respir J. 2013;42 Suppl 57:P2230.

64. Alahmari AD, Kowlessar BS, Patel AR, et al. Physical activity and exercise capacity in patients with moderate COPD exacerbations. Eur Respir J. 2016;48(2):340-349.

65. Garcia-Polo C, Alcazar-Navarrete B, Ruiz-Iturriaga LA, et al. Factors associated with high healthcare resource utilisation among COPD patients. Respir Med. 2012;106(12):1734-1742.

66. Ghanbari BH, Garland SJ, Road JD, Reid WD. Pain and physical performance in people with COPD. Respir Med. 2013;107(11):1692-1699.

67. Akor AA, Obaseki D, Adeniyl B, et al. COPD assessment test in patients with COPD in Africa: a preliminary survey. Am J Respir Crit Care Med. 2014;189:A2951.

68. Ijiri N, Kanazawa H, Yoshikawa T, Hirata K. Application of a new parameter in the 6-minute walk test for manifold analysis of exercise capacity in patients with COPD. Int J Chron Obstruct Pulmon Dis. 2014;9:1235-1240.

69. Bell M, Fotheringham I, Punekar YS, Riley JH, Cockle S, Singh SJ. Systematic review of the association between laboratory- and field based exercise tests and lung function in patients with chronic obstructive pulmonary disease. Chronic Obstr Pulm Dis. 2015;2(3):321-342.

70. National Institute for Health and Care Excellence. Management of Chronic Obstructive Pulmonary Disease in Adults in Primary and Secondary Care. Partial update. London: NICE; 2010.

71. American Thoracic Society/European Respiratory Society Task Force. Standards for the Diagnosis and Management of Patients with COPD. Version 1.2. New York: American Thoracic Society; 2005.

72. Celli BR, Cote CG, Marin JM, et al. The body-mass index, airflow obstruction, dyspnea, and exercise capacity index in chronic obstructive pulmonary disease. $N$ Engl J Med. 2004;350(10):1005-1012.

73. Ong KC, Earnest A, Lu SJ. A multidimensional grading system (BODE index) as predictor of hospitalization for COPD. Chest. 2005;128(6): 3810-3816.

74. [No authors listed]. Rationale and design of the national emphysema treatment trial: a prospective randomized trial of lung volume reduction surgery. Chest. 1999;116(6):1750-1761.

75. Fishman A, Martinez F, Naunheim K, et al. A randomized trial comparing lung-volume-reduction surgery with medical therapy for severe emphysema. N Eng J Med. 2003;348(21):2059-2073.

76. Sassi-Dambron DE, Eakin EG, Ries AL, Kaplan RM. Treatment of dyspnea in COPD: a controlled clinical trial of dyspnea management strategies. Chest. 1995;107(3):724-729.

This journal is indexed on PubMed Central, MedLine and CAS. The manuscript management system is completely online and includes a very quick and fair peer-review system, which is all easy to use. Visit http://www.dovepress.com/testimonials.php to read real quotes from published authors. 